\title{
Measurements of low- $p_{\mathrm{T}}$ electrons from semileptonic heavy-flavour hadron decays at mid-rapidity in pp and $\mathrm{Pb}-\mathrm{Pb}$ collisions at $\sqrt{s_{\mathrm{NN}}}=2.76 \mathrm{TeV}$
}

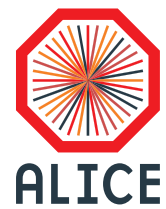

\section{The ALICE collaboration}

E-mail: ALICE-publications@cern.ch

AbStRact: Transverse-momentum $\left(p_{\mathrm{T}}\right)$ differential yields of electrons from semileptonic heavy-flavour hadron decays have been measured in the most central $(0-10 \%)$ and in semicentral $(20-40 \%) \mathrm{Pb}-\mathrm{Pb}$ collisions at $\sqrt{s_{\mathrm{NN}}}=2.76 \mathrm{TeV}$. The corresponding production cross section in pp collisions has been measured at the same energy with substantially reduced systematic uncertainties with respect to previously published results. The modification of the yield in $\mathrm{Pb}-\mathrm{Pb}$ collisions with respect to the expectation from an incoherent superposition of nucleon-nucleon collisions is quantified at mid-rapidity $(|y|<0.8)$ in the $p_{\mathrm{T}}$ interval $0.5-3 \mathrm{GeV} / c$ via the nuclear modification factor, $R_{\mathrm{AA}}$. This paper extends the $p_{\mathrm{T}}$ reach of the $R_{\mathrm{AA}}$ measurement towards significantly lower values with respect to a previous publication. In $\mathrm{Pb}-\mathrm{Pb}$ collisions the $p_{\mathrm{T}}$-differential measurements of yields at low $p_{\mathrm{T}}$ are essential to investigate the scaling of heavy-flavour production with the number of binary nucleon-nucleon collisions. Heavy-quark hadronization, a collective expansion and even initial-state effects, such as the nuclear modification of the Parton Distribution Function, are also expected to have a significant effect on the measured distribution.

KEYWORDS: Heavy Ion Experiments

ArXiv EPrint: 1805.04379 


\section{Contents}

1 Introduction 1

2 Experimental apparatus and data sample 3

3 Data analysis $\quad \mathbf{5}$

3.1 Track selection and electron identification 5

$\begin{array}{lll}3.2 & \text { Subtraction of electrons from non heavy-flavour sources } & 6\end{array}$

3.3 Correction and normalisation 8

3.4 Systematic uncertainties 9

4 Results 10

$\begin{array}{lll}4.1 & p_{\mathrm{T}} \text {-differential invariant cross section in pp collisions } & 10\end{array}$

$\begin{array}{lll}4.2 & p_{\mathrm{T}} \text {-differential invariant yields in } \mathrm{Pb}-\mathrm{Pb} \text { collisions } & 11\end{array}$

$\begin{array}{lll}4.3 & \text { Nuclear modification factor } R_{\mathrm{AA}} & 12\end{array}$

5 Comparison with model calculations $\quad 13$

$\begin{array}{llr}6 & \text { Conclusions } & 14\end{array}$

$\begin{array}{ll}\text { The ALICE collaboration } & 23\end{array}$

\section{Introduction}

In ultra-relativistic heavy-ion collisions at the Relativistic Heavy-Ion Collider (RHIC) and at the Large Hadron Collider (LHC), strongly-interacting matter characterized by high energy density and temperature is produced [1-6]. Under these conditions, the formation of a deconfined state of quarks and gluons, called Quark-Gluon Plasma (QGP), is predicted by Quantum ChromoDynamic (QCD) calculations on the lattice [7-11]. The production of heavy quarks, i.e. charm (c) and beauty (b), takes place via initial partonic scattering processes with large momentum transfer (hard scattering) on a timescale of $\hbar /\left(2 m_{\mathrm{c}, \mathrm{b}} c^{2}\right)$, where $m$ is the mass of the quark. This timescale (e.g. $\approx 0.08 \mathrm{fm} / c$ for charm) is smaller than the QGP thermalization time $(\approx 0.6-1 \mathrm{fm} / c[12])$. Additional thermal production as well as annihilation rates of charm and beauty quarks in the strongly interacting medium are expected to be small in $\mathrm{Pb}-\mathrm{Pb}$ collisions even at LHC energies [13-15]. Consequently, charm and beauty quarks are ideal probes to investigate the properties of the QGP, since they experience the full evolution of the strongly interacting medium produced in highenergy heavy-ion collisions.

In order to exploit the sensitivity of heavy-flavour observables to medium effects a precise reference where such effects are not expected is needed and it is provided by pp 
collisions. In pp collisions, heavy-quark production can be described theoretically via perturbative QCD calculations over the full quark momentum range, while such a description does not hold for gluon and light-quark production [13]. Therefore, measurements of heavy-flavour production cross sections in pp collisions are used to test perturbative QCD calculations and provide the necessary experimental reference for heavy-ion collisions.

The modification of the $p_{\mathrm{T}}$-differential yield in heavy-ion collisions with respect to $\mathrm{pp}$ collisions at the same centre-of-mass energy is quantified by the nuclear modification factor $R_{\mathrm{AA}}$, defined as:

$$
R_{\mathrm{AA}}\left(p_{\mathrm{T}}, y\right)=\frac{1}{\left\langle T_{\mathrm{AA}}\right\rangle} \cdot \frac{\mathrm{d}^{2} N_{\mathrm{AA}} / \mathrm{d} p_{\mathrm{T}} \mathrm{d} y}{\mathrm{~d}^{2} \sigma_{\mathrm{pp}} / \mathrm{d} p_{\mathrm{T}} \mathrm{d} y}
$$

where $\mathrm{d}^{2} N_{\mathrm{AA}} / \mathrm{d} p_{\mathrm{T}} \mathrm{d} y$ is the yield measured in heavy-ion collisions in a given $p_{\mathrm{T}}$ and $y$ interval, and $\mathrm{d}^{2} \sigma_{\mathrm{pp}} / \mathrm{d} p_{\mathrm{T}} \mathrm{d} y$ is the corresponding production cross section in pp collisions. The average nuclear overlap function, $\left\langle T_{\mathrm{AA}}\right\rangle$, is given by the ratio of the average number of binary nucleon-nucleon collisions in a centrality class and the inelastic nucleon-nucleon cross section, and it is determined via Glauber model calculations $[16,17]$. In the absence of medium effects, $R_{\mathrm{AA}}$ is expected to be unity for hard probes such as charm and beauty production.

For momenta larger than the masses of charm and beauty quarks, the dominant medium effect is the partonic energy loss via radiative $[18,19]$ and collisional processes $[20-$ 22 ] when heavy quarks propagate through the QGP. These processes are expected to cause a shift of the partonic momentum distribution towards lower momenta and, therefore, to lead to a suppression of the yield of heavy-flavour hadrons and their decay products at high $p_{\mathrm{T}}(\gtrsim 2 \mathrm{GeV} / c)$ and, consequently, to $R_{\mathrm{AA}}<1$. In the absence of further processes that modify the total charm and/or beauty production cross section or the fragmentation/hadronization of heavy quarks, $R_{\mathrm{AA}}$ is expected to increase again towards low $p_{\mathrm{T}}$ to compensate the suppression at high $p_{\mathrm{T}}$ and, therefore, conserve the binary collision scaling. At RHIC, such a rise was observed by the PHENIX and STAR experiments for leptons from semileptonic heavy-flavour hadron decays in $\mathrm{Au}-\mathrm{Au}$ and $\mathrm{Cu}-\mathrm{Cu}$ collisions at $\sqrt{s_{\mathrm{NN}}}=200 \mathrm{GeV}[23-26]$. The STAR Collaboration also measured the $R_{\mathrm{AA}}$ of $\mathrm{D}^{0}$ mesons in $\mathrm{Au}-\mathrm{Au}$ collisions for $p_{\mathrm{T}}<8 \mathrm{GeV} / c[27]$.

The interaction of charm and, to a lesser extent, beauty quarks of low transverse momentum with the medium may lead to the participation of heavy quarks in the collective expansion of the hot and dense system $[28,29]$ and, eventually, to a partial or complete thermalization of heavy quarks in the system [30]. Moreover, while in pp collisions charm and beauty quarks hadronize via fragmentation, in heavy-ion collisions a competing hadronization mechanism through the coalescence with other quarks from the medium could become relevant and modify the phase-space distribution of heavy-flavour hadrons up to transverse momenta of a few $\mathrm{GeV} / c$ [31-33]. Finally, initial-state effects due the presence of a heavy nucleus in the collision system can play a role. At low Bjorken- $x$ (below $10^{-2}$ ) the parton densities in nucleons bounded in nuclei are reduced with respect to those in free nucleons. This so-called "shadowing" leads to a reduction of heavy-flavour production, becoming more pronounced with decreasing $p_{\mathrm{T}}$ [34]. In addition, at lower collision energies, momen- 
tum $\left(k_{\mathrm{T}}\right)$ broadening leads to an enhancement of $R_{\mathrm{AA}}$ at intermediate $p_{\mathrm{T}}$, the so-called Cronin effect [35].

At the LHC, open heavy-flavour production was measured in $\mathrm{Pb}-\mathrm{Pb}$ collisions via exclusive hadron decays of prompt $\mathrm{D}$ and $\mathrm{B}$ mesons and via leptons from heavy-flavour hadron decays [36-43]. At high $p_{\mathrm{T}}(\gtrsim 3 \mathrm{GeV} / c)$, a substantial suppression with respect to the scaled reference cross section from pp collisions is observed with $R_{\mathrm{AA}}$ values similar to those measured at RHIC. At lower $p_{\mathrm{T}}$, the $R_{\mathrm{AA}}$ of prompt $\mathrm{D}$ mesons stays below unity down to transverse momenta as low as $1 \mathrm{GeV} / c$, in contrast to corresponding measurements at RHIC where $R_{\mathrm{AA}}$ reaches a maximum value of $\approx 1.5$ at $p_{\mathrm{T}} \approx 1-2 \mathrm{GeV} / c$. The different patterns observed at the LHC and at RHIC could be due to differences in the initial momentum distributions of heavy quarks, the magnitude of parton energy loss in the medium, the impact of collective expansion, the relevance of coalescence as a hadronization mechanism, and the role of initial-state effects [43].

At the LHC, initial-state effects and their impact on the nuclear modification factor are investigated in proton-lead ( $\mathrm{p}-\mathrm{Pb}$ ) collisions. The nuclear modification factor $R_{\mathrm{pPb}}$ was measured at mid-rapidity for prompt $\mathrm{D}$ and $\mathrm{B}$ mesons and for electrons from semileptonic heavy-flavour hadron decays $[38,44-46]$. The $R_{\mathrm{pPb}}$ of electron from heavy-flavour hadron decay was observed to be consistent with unity within uncertainties over the whole $p_{\mathrm{T}}$ range of the measurements, as expected from binary-collision scaling of heavy-flavour production.

This paper reports on measurements of electrons from semileptonic heavy-flavour hadron decays at mid-rapidity $(|y|<0.8)$ in pp collisions at $\sqrt{s}=2.76 \mathrm{TeV}$ and in $\mathrm{Pb}-\mathrm{Pb}$ collisions in the two centrality classes $0-10 \%$ and $20-40 \%$ at $\sqrt{s_{\mathrm{NN}}}=2.76 \mathrm{TeV}$. The charge averaged $p_{\mathrm{T}}$-differential yields, cross sections and the resulting nuclear modification factors are presented. Applying a data-driven background subtraction technique [45] allowed for a reduction of the systematic uncertainties of the pp reference cross section by a factor of about 3 compared to the previously published reference [47], which is consistent within uncertainties with the current measurement.

The results presented in this paper extend the previous measurements [42] of electrons from semileptonic heavy-flavour hadron decays in $\mathrm{Pb}-\mathrm{Pb}$ collisions from $3 \mathrm{GeV} / c$ down to $0.5 \mathrm{GeV} / c$ in $p_{\mathrm{T}}$. They complement the measurements of muons from semileptonic heavyflavour hadron decays at forward rapidity and of the prompt D mesons at mid-rapidity reported by the ALICE Collaboration [39, 41], as well as of muons from semileptonic heavy-flavour hadron decays at mid-rapidity reported by the ATLAS Collaboration [48]. The measured nuclear modification factor $R_{\mathrm{AA}}$ is compared with model calculations aiming at describing heavy-quark production and energy loss in heavy-ion collisions taking into account also initial-state effects.

\section{Experimental apparatus and data sample}

The ALICE apparatus, described in detail in [49, 50], consists mainly of a central barrel at mid-rapidity $(|\eta|<0.9)$ embedded in a solenoidal magnet, and a muon spectrometer at forward rapidity $(-4<\eta<-2.5)$. In the following, the subsystems which are used to perform the measurement of electrons from heavy-flavour hadron decays are described. 
Charged-particle tracks are reconstructed at mid-rapidity $(|\eta|<0.9)$ with the Inner Tracking System (ITS) and the Time Projection Chamber (TPC). The ITS [51] consists of six cylindrical silicon layers surrounding the beam vacuum pipe. The first two layers, made of Silicon Pixel Detectors (SPD) to cope with the high particle density in the proximity of the interaction point, provide an excellent position resolution of $12 \mu \mathrm{m}$ and $100 \mu \mathrm{m}$ in the $r \varphi$ and the beam direction ( $z$-coordinate of the reference system), respectively. The third and fourth layers consist of Silicon Drift Detectors (SDD), while the two outermost layers are made of Silicon Strip Detectors (SSD). The SDD and SSD layers are also used for charged-particle identification via energy loss $(\mathrm{d} E / \mathrm{d} x)$ measurements.

The TPC [52] is the main tracking detector in the central barrel and provides a chargedparticle momentum measurement together with excellent two-track separation and particle identification via $\mathrm{d} E / \mathrm{d} x$ determination.

The Time-Of-Flight (TOF) detector [53] provides the measurement of the time-of-flight for charged particles from the interaction point up to the detector radius of $3.8 \mathrm{~m}$, with an overall resolution of about $80 \mathrm{ps}$. The measured time-of-flight of electrons is well separated from that of kaons and protons up to $p_{\mathrm{T}} \simeq 2.5 \mathrm{GeV} / c$ and $p_{\mathrm{T}} \simeq 4 \mathrm{GeV} / c$, respectively.

The V0 detectors [54] consist of two arrays of 32 scintillator tiles covering the pseudorapidity ranges $2.8<\eta<5.1$ (V0A) and $-3.7<\eta<-1.7$ (V0C), respectively, and are used for triggering and for centrality estimation. The latter is performed through a Glauber Monte Carlo (MC) fit of the signal amplitude in the two scintillator detectors [5557]. Together with the Zero Degree Calorimeters (ZDC) [58], located on both sides of the interaction point at $\mathrm{z} \approx \pm 114 \mathrm{~m}$, they are used offline for event selection.

The pp results presented in this paper are based on the same minimum-bias (MB) data sample recorded at $\sqrt{s}=2.76 \mathrm{TeV}$ as the previously published result [47]. The MB trigger required at least one hit in the SPD or a signal (above threshold) in either of the two V0 arrays, in temporal coincidence with a signal from the beam position monitors [50]. Pile-up events are identified and rejected using the SPD [47, 59], and they amount to about $0.7 \%$ of all events. During the pp run at $2.76 \mathrm{TeV}$, the information from the SDD was read out only for a fraction of the recorded events to maximize the data acquisition speed. For the current analysis all events have been reconstructed without the SDD information in order to obtain a homogeneous sample over the full statistics.

For the $\mathrm{Pb}-\mathrm{Pb}$ analysis, the same data sample recorded at $\sqrt{s_{\mathrm{NN}}}=2.76 \mathrm{TeV}$ was used as for previous publications $[28,42]$. The events were collected with a MB interaction trigger using information from the coincidence of signals between the V0A and V0C detectors. Central and semi-central $\mathrm{Pb}-\mathrm{Pb}$ collisions were selected online by applying different thresholds on the V0 signal amplitudes resulting in central (0-10\%) and semi-central (10$50 \%)$ trigger classes [50]. Events affected by pile-up from different bunch crossings have been rejected offline [28]. This selection removes up to $5 \%$ of the total number of events depending on the centrality of the collisions.

For both collision systems, only events with a reconstructed interaction vertex (primary vertex) within $10 \mathrm{~cm}$ from the nominal interaction point along the beam direction are used in order to minimize edge effects at the limit of the central barrel acceptance. The number of events analysed after applying the event selection and the corresponding luminosities 


\begin{tabular}{|ccc|}
\hline Collision system & $N_{\text {events }}$ & $\left\langle T_{\mathrm{AA}}\right\rangle\left(\mathrm{mb}^{-1}\right)$ \\
\hline $\mathrm{pp}$ & $38.9 \times 10^{6}$ & - \\
\hline $\mathrm{Pb}-\mathrm{Pb}, 0-10 \%$ & $15.4 \times 10^{6}$ & $23.37 \pm 0.2$ \\
$\mathrm{~Pb}-\mathrm{Pb}, 20-40 \%$ & $8.2 \times 10^{6}$ & $7.109 \pm 0.15$ \\
\hline
\end{tabular}

Table 1. Number of events for the pp collisions and the two $\mathrm{Pb}-\mathrm{Pb}$ centrality classes after applying the event selection. In the right column the average nuclear overlap function is reported for the $\mathrm{Pb}-\mathrm{Pb}$ samples [60].

for the pp and the two $\mathrm{Pb}-\mathrm{Pb}$ centrality classes are listed in table 1 . The values of the average nuclear overlap function for the two $\mathrm{Pb}-\mathrm{Pb}$ centrality classes are listed as well. These values and the respective uncertainties are updated with respect to the previously published high- $p_{\mathrm{T}} R_{\mathrm{AA}}$ results [42]. More information about the update of the average nuclear overlap function values can be found in [60].

\section{Data analysis}

The $p_{\mathrm{T}}$-differential yield of electrons from semileptonic heavy-flavour hadron decays is computed by measuring the inclusive electron yield and subtracting the contribution of electrons that do not originate from open heavy-flavour hadron decays. In the following, the inclusive electron identification strategy and the subtraction of electrons originating from background sources are described for the analysis of $\mathrm{pp}$ and $\mathrm{Pb}-\mathrm{Pb}$ collisions.

\subsection{Track selection and electron identification}

Candidate electrons tracks are required to fulfil the criteria summarized in table 2, similarly to what was done in refs. $[28,47]$, in order to select good quality tracks. The rapidity range used in the analyses is restricted to $|y|<0.8$ to exclude the edges of the detectors, where the systematic uncertainties related to particle identification increase.

The electron identification is mainly based on the measurement of the specific ionization energy loss in the TPC $(\mathrm{d} E / \mathrm{d} x)$, similarly to the procedure followed in refs. [28, 47]. The discriminant variable is the deviation of $\mathrm{d} E / \mathrm{d} x$ from the parametrized electron BetheBloch [61] expectation value, expressed in units of the $\mathrm{d} E / \mathrm{d} x$ resolution, $n_{\sigma}^{\mathrm{TPC}}$ [50].

In order to reduce the hadron contamination in $\mathrm{Pb}-\mathrm{Pb}$ collisions, tracks with a timeof-flight differing from the expected value for electrons $\left(n_{\sigma}^{\mathrm{TOF}}\right)$ by twice the TOF resolution or more are rejected. In pp collisions, a $\left|n_{\sigma}^{\mathrm{TOF}}\right| \geq 3$ rejection is applied due to the smaller hadron contamination.

In $\mathrm{Pb}-\mathrm{Pb}$ collisions, in addition, the $\mathrm{d} E / \mathrm{d} x$ in the ITS is used to further reject hadrons. To guarantee a good Particle IDentification (PID) based on the $\mathrm{d} E / \mathrm{d} x$ in the ITS, tracks are required to have at least three out of the four possible hits in the external layers of the ITS (SDD and SSD), which can provide $\mathrm{d} E / \mathrm{d} x$ measurements. Table 3 summarizes the PID selection criteria for electron identification.

The remaining hadron contamination is estimated by fitting in momentum slices the TPC $\mathrm{d} E / \mathrm{d} x$ distribution after the TOF (and ITS) PID selections $[28,59]$. The hadron 


\begin{tabular}{|ccc|}
\hline Data Sample & $\mathrm{Pb}-\mathrm{Pb}$ & $\mathrm{pp}$ \\
\hline$p_{\mathrm{T}}$ range $(\mathrm{GeV} / c)$ & $0.5-3$ & $0.5-3$ \\
$|y|$ & $<0.8$ & $<0.8$ \\
\hline Number of TPC clusters & $\geq 100$ & $\geq 110$ \\
Number of TPC clusters in $\mathrm{d} E / \mathrm{d} x$ calculation & $\geq 90$ & $\geq 80$ \\
Ratio of found TPC clusters over findable & $>0.6$ & $>0.6$ \\
$\chi^{2} /$ clusters of the momentum fit in the TPC & $<3.5$ & $<4$ \\
DCA $_{x y}$ & $<2.4 \mathrm{~cm}$ & $<1 \mathrm{~cm}$ \\
DCA $_{z}$ & $<3.2 \mathrm{~cm}$ & $<2 \mathrm{~cm}$ \\
Number of ITS hits & $\geq 5$ & $\geq 3$ \\
Number of hits in the SPD layers & 2 & 2 \\
\hline
\end{tabular}

Table 2. Track selection criteria used in the analyses. DCA is an abbreviation for the distance of closest approach of a track to the primary vertex.

\begin{tabular}{|ccccc|}
\hline & $p_{\mathrm{T}}$ range $(\mathrm{GeV} / c)$ & $\begin{array}{c}\mathrm{TPC} \mathrm{d} E / \mathrm{d} x \\
\text { selection }\end{array}$ & $\begin{array}{c}\text { ITS } \mathrm{d} E / \mathrm{d} x \\
\text { selection }\end{array}$ & $\begin{array}{c}\text { TOF compatibility } \\
\text { with } e \text { hypothesis }\end{array}$ \\
\hline $\mathrm{pp}$ & $0.5-3$ & $-1<n_{\sigma}^{\mathrm{TPC}}<3$ & - & $\left|n_{\sigma}^{\mathrm{TOF}}\right|<3$ \\
\hline $\mathrm{Pb}-\mathrm{Pb}$ & $0.5-1.5$ & $-1<n_{\sigma}^{\mathrm{TPC}}<3$ & $\left|n_{\sigma}^{\mathrm{ITS}}\right|<1$ & $\left|n_{\sigma}^{\mathrm{TOF}}\right|<2$ \\
& $1.5-3$ & $0<n_{\sigma}^{\mathrm{TPC}}<3$ & $\left|n_{\sigma}^{\mathrm{ITS}}\right|<2$ & $\left|n_{\sigma}^{\mathrm{TOF}}\right|<2$ \\
\hline
\end{tabular}

Table 3. Electron identification criteria used in the analyses (see text for more details).

contamination is negligible at the lowest $p_{\mathrm{T}}$ and it increases with $p_{\mathrm{T}}$, reaching about $5 \%$ at $p_{\mathrm{T}}=3 \mathrm{GeV} / c$ in $\mathrm{Pb}-\mathrm{Pb}$ collisions and about $1 \%$ in pp collisions, with negligible dependence on centrality and pseudorapidity. In both collision systems the hadron contamination is subtracted statistically from the inclusive electron candidate yield.

\subsection{Subtraction of electrons from non heavy-flavour sources}

The raw inclusive sample of electron candidates $\left(p_{\mathrm{T}}<3 \mathrm{GeV} / c\right)$ consists of the signal, i.e. the electrons from semileptonic heavy-flavour hadron decays, and four background components:

1. photonic electrons from Dalitz decays of light neutral mesons (predominantly $\pi^{0}$ and $\eta$ mesons) and the conversion of their decay photons in the detector material, as well as from prompt virtual and real photons from thermal and hard scattering processes;

2. electrons from weak $\mathrm{K}^{0 / \pm} \rightarrow \mathrm{e}^{ \pm} \pi^{\mp / 0} \stackrel{(-)}{\nu_{e}}\left(\mathrm{~K}_{\mathrm{e} 3}\right)$ decays;

3. dielectron decays of quarkonia;

4. dielectron decays of light vector mesons.

The photonic-electron tagging method $[45,62]$ is adopted for the subtraction of the first and main background component. For $p_{\mathrm{T}}<1.5 \mathrm{GeV} / c$ the inclusive electron yield 


\begin{tabular}{|ccc|}
\hline Associated electron & $\mathrm{Pb}-\mathrm{Pb}$ & $\mathrm{pp}$ \\
\hline$p_{\mathrm{T}}(\mathrm{GeV} / c)$ & $>0.15$ & $>0.1$ \\
$|y|$ & $<0.9$ & $<0.8$ \\
Number of TPC clusters & $\geq 80$ & $\geq 60$ \\
Number of ITS hits & $\geq 2$ & $\geq 2$ \\
$\mathrm{DCA}_{x y}$ & $<2.4 \mathrm{~cm}$ & $<1 \mathrm{~cm}$ \\
$\mathrm{DCA}_{z}$ & $<3.2 \mathrm{~cm}$ & $<2 \mathrm{~cm}$ \\
TPC $\mathrm{d} E / \mathrm{d} x$ & $\left|n_{\sigma}^{\mathrm{TPC}}\right|<3$ & $\left|n_{\sigma}^{\mathrm{TPC}}\right|<3$ \\
\hline Electron-positron pair & & \\
\hline$m_{\mathrm{e}^{+}} \mathrm{e}^{-}\left(\mathrm{MeV} / c^{2}\right)$ & $<70$ & $<140$ \\
\hline
\end{tabular}

Table 4. Selection criteria for tagging photonic electrons in $\mathrm{Pb}-\mathrm{Pb}$ and $\mathrm{pp}$ collisions.

is largely dominated by the contribution of photonic electrons. The ratio of the signal to the photonic electron background is measured to be 0.2 at $p_{\mathrm{T}}=0.5 \mathrm{GeV} / c$ and it is observed to increase reaching a value of 3 at $p_{\mathrm{T}}=3 \mathrm{GeV} / c$ [28]. Photonic electrons originate from electron-positron pairs with a small invariant mass $\left(m_{\mathrm{e}^{+} \mathrm{e}^{-}}\right)$. They are tagged by pairing an electron (positron) track with opposite charge tracks identified as positrons (electrons) from the same event. The latter are called associated electrons in the following and they are selected with less stringent requirements listed in table 4 . The combinatorial background from uncorrelated electron-positron pairs is subtracted using as a proxy the like-sign invariant mass distribution in the same invariant mass interval. A selection on the pair invariant mass is applied as listed in table 4 .

Due to detector acceptance and inefficiencies and because of the decay kinematics, not all photonic electrons in the inclusive electron sample are tagged with this method. Therefore, the raw yield of tagged photonic electrons is corrected for the efficiency to find the associated electron (positron), hereafter called tagging efficiency. This efficiency is estimated with Monte Carlo (MC) simulations. In particular, HIJING v1.383 [63] was used to simulate $\mathrm{Pb}-\mathrm{Pb}$ collisions, while the PYTHIA 6 (Perugia 2011 tune) [64] event generator was used for the simulation of pp events. The transport of particles in the detector is performed using GEANT3 [65]. In both analyses, the generated $\pi^{0} p_{\mathrm{T}}$ distributions in $\mathrm{MC}$ are weighted so as to match the measured neutral pion $p_{\mathrm{T}}$ spectra $[66,67]$. In the pp analysis, the $\eta p_{\mathrm{T}}$ spectra are weighted using the corresponding measurement [68], while for $\mathrm{Pb}-\mathrm{Pb}$ collisions the $\eta$ weights are determined via $m_{\mathrm{T}}$-scaling of the measured $\pi^{0} p_{\mathrm{T}}$ spectra $[69,70]$. The resulting $\eta / \pi^{0}$ ratios agree within uncertainties with the ratios measured by ALICE in $0-10 \%$ and $20-50 \%$ central $\mathrm{Pb}-\mathrm{Pb}$ collisions at $\sqrt{s_{\mathrm{NN}}}=$ $2.76 \mathrm{TeV}$ [71]. The photonic electron tagging efficiency increases with the electron $p_{\mathrm{T}}$, starting from a value of $\approx 40 \%(\approx 30 \%)$ at $p_{\mathrm{T}}=0.5 \mathrm{GeV} / c$ and reaching a value of $\approx 70 \%$ $(\approx 60 \%)$ at $p_{\mathrm{T}}=3 \mathrm{GeV} / c$ for pp $(\mathrm{Pb}-\mathrm{Pb})$ collisions.

The background contribution of non-photonic electrons from $\mathrm{K}_{\mathrm{e} 3}$ decays and the dielectron decay of $\mathrm{J} / \psi$ mesons is subtracted from the fully corrected and normalized electron yield using the so-called cocktail approach in both pp and $\mathrm{Pb}-\mathrm{Pb}$ collisions [24, 45, 47, 59]. 
Due to the requirement of hits in both pixel layers, the relative contribution from $\mathrm{K}_{\mathrm{e} 3}$ decays to the electron background is small and it decreases with $p_{\mathrm{T}}$, with a maximum of about $0.5 \%$ at $p_{\mathrm{T}}=0.5 \mathrm{GeV} / c$ for both the collision systems. For pp collisions, the contribution of electrons from $\mathrm{J} / \psi$ decays is calculated based on a phenomenological interpolation of the $\mathrm{J} / \psi$ production cross sections measured at various values of $\sqrt{s}$ as described in [72], and as done in a previous analysis [47]. For $\mathrm{Pb}-\mathrm{Pb}$ collisions, the $p_{\mathrm{T}}$-differential $\mathrm{J} / \psi$ yield is calculated by multiplying this reference $\mathrm{J} / \psi$ cross section in pp collisions with $\left\langle T_{\mathrm{AA}}\right\rangle$ and the measured nuclear modification factor in $\mathrm{Pb}-\mathrm{Pb}$ collisions $[73,74]$. The contribution of electrons from $\mathrm{J} / \psi$ decays is maximal in the interval $2.0<p_{\mathrm{T}}<3.0 \mathrm{GeV} / c$, with a value of $\approx 3 \%$ in pp collisions and of $\approx 5 \%$ in central $\mathrm{Pb}-\mathrm{Pb}$ collisions. At higher $p_{\mathrm{T}}$ and in less central $\mathrm{Pb}-\mathrm{Pb}$ collisions the background from $\mathrm{J} / \psi$ decays decreases. At lower $p_{\mathrm{T}}$ it is negligible. The background from dielectron decays of light vector mesons and other quarkonium states as well as from Dalitz decays of higher mass mesons $\left(\omega, \eta^{\prime}, \phi\right)$ is negligible as discussed in ref. [28].

\subsection{Correction and normalisation}

After the statistical subtraction of the hadron contamination and the background from photonic electrons, the raw yield of electrons and positrons is divided by the number of events analysed $\left(N_{\mathrm{ev}}^{M B}\right)$, by the value of $p_{\mathrm{T}}$ at the centre of each bin and its width $\Delta p_{\mathrm{T}}$, by the width $\Delta y$ of the covered rapidity interval, by the geometrical acceptance $\left(\epsilon^{\text {geo }}\right)$ times the reconstruction $\left(\epsilon^{\mathrm{reco}}\right)$ and PID efficiencies $\left(\epsilon^{\mathrm{eID}}\right)$ and a factor of two to obtain the charge averaged invariant differential yield

$$
\frac{1}{2 \pi p_{\mathrm{T}}} \frac{\mathrm{d}^{2} N^{e^{ \pm}}}{\mathrm{d} p_{\mathrm{T}} \mathrm{d} y}=\frac{1}{2} \frac{1}{2 \pi p_{\mathrm{T}, \text { centre }}} \frac{1}{N_{\mathrm{ev}}^{M B}} \frac{1}{\Delta y \Delta p_{\mathrm{T}}} \frac{N_{\text {raw }}^{e^{ \pm}}\left(p_{\mathrm{T}}\right)}{\left(\epsilon^{\text {geo }} \times \epsilon^{\mathrm{reco}} \times \epsilon^{\mathrm{eID}}\right)} .
$$

The invariant production cross section in pp collisions is obtained by further multiplying with the minimum-bias trigger cross section for pp collisions at $\sqrt{s}=2.76 \mathrm{TeV}, \sigma_{\mathrm{MB}}=$ $(55.4 \pm 1.0) \mathrm{mb}[75]$.

The efficiencies are determined using dedicated MC simulations. The reconstruction efficiencies are computed using a heavy-flavour enriched PYTHIA 6 [64] MC sample in which each simulated pp event contains a $c \bar{c}$ or $b \bar{b}$ pair, and heavy-flavour hadrons are forced to decay semi-electronically. In the $\mathrm{MC}$ production used for the $\mathrm{Pb}-\mathrm{Pb}$ analysis the underlying events are simulated using the HIJING v1.383 generator [63] and the heavyflavour signal from the PYTHIA 6 generator is added. Out of all produced particles in these PYTHIA pp events, only the heavy-flavour decay products are kept and transported through the detector together with the particles produced with HIJING. In order to better reproduce the experimental conditions for the detector occupancy, the number of heavy quarks injected into each HIJING event is adjusted according to the $\mathrm{Pb}-\mathrm{Pb}$ collision centrality. In $\mathrm{Pb}-\mathrm{Pb}$ collisions, the bin-wise total reconstruction efficiencies $\left(\epsilon^{\mathrm{geo}} \times \epsilon^{\mathrm{reco}} \times\right.$ $\left.\epsilon^{\mathrm{eID}}\right)$ do not show any significant $p_{\mathrm{T}}$ dependence and are about $8 \%(9 \%)$ in the $0-10 \%$ (20-40\%) centrality class. Due to the less stringent selections applied for pp collisions, the total electron reconstruction efficiency reaches a value of about $27 \%$ at $p_{\mathrm{T}}=3 \mathrm{GeV} / c$ in this case. Finally, the remaining background contributions from weak $\mathrm{K}_{\mathrm{e} 3}$ decays and 
dielectron decays of $\mathrm{J} / \psi$ mesons are subtracted from the fully corrected cross section (yield) for $\mathrm{pp}(\mathrm{Pb}-\mathrm{Pb})$ collisions.

\subsection{Systematic uncertainties}

The overall systematic uncertainty on the $p_{\mathrm{T}}$ spectra is calculated summing in quadrature the different uncorrelated contributions, which are summarised in table 5 and discussed in the following.

The systematic uncertainties arising from the residual discrepancy between MC used to determine the total reconstruction efficiency and data is estimated by systematically varying the track selection and PID requirements around the default values chosen in the analysis. The systematic uncertainties are determined as the root mean squared (RMS) of the distribution of the resulting corrected yields (or cross sections in pp) obtained for different selections in each $p_{\mathrm{T}}$ interval, considering also shifts of the mean value with respect to the default selections. In the $\mathrm{Pb}-\mathrm{Pb}$ analysis, this contribution is about $6 \%$ at low $p_{\mathrm{T}}$ $\left(p_{\mathrm{T}}<1 \mathrm{GeV} / c\right)$, and it decreases with increasing $p_{\mathrm{T}}$ reaching about $3 \%$ at the highest $p_{\mathrm{T}}$. In the pp case this contribution is about $4 \%$ without $p_{\mathrm{T}}$ dependence.

In the pp analysis, a systematic uncertainty of about $2 \%(3 \%)$ is assigned due to the incomplete knowledge of the efficiency in matching tracks reconstructed in the ITS and TPC (TPC and TOF) $[47,59]$. In $\mathrm{Pb}-\mathrm{Pb}$ collisions, the uncertainty assigned on the measurements coming from the track-reconstruction procedure amounts to $5 \%$ for single tracks [76].

The solenoid polarity was changed during the $\mathrm{Pb}-\mathrm{Pb}$ data taking period. From the comparison of the fully corrected spectra of electrons from semileptonic heavy-flavour hadron decays measured in events with the magnetic field oriented in the two opposite directions, a $2 \%$ systematic uncertainty is assigned for $p_{\mathrm{T}} \leq 1.25 \mathrm{GeV} / c$. To ensure that the results are not biased by tracks detected at the edges of the detector, where the efficiencies are more difficult to be calculated, the measurements were re-done restricting the rapidity window for the electrons down to $|y|<0.5$. In addition, possible biases in the efficiency determination are checked by performing the analyses only in the positive or the negative rapidity region. A $5 \%$ systematic uncertainty has been estimated for $p_{\mathrm{T}}<1.5 \mathrm{GeV} / c$ in both pp and $\mathrm{Pb}-\mathrm{Pb}$ collisions.

The systematic uncertainty arising from the photonic-electron subtraction technique is estimated similarly as the RMS of the distribution of yields obtained by varying the selection criteria listed in table 4 . In the $\mathrm{Pb}-\mathrm{Pb}$ analysis, because of the large combinatorial background of random pairs, this systematic uncertainty is of the order of $\pm 30 \%$ in the $0-10 \%$ most-central collisions and $\pm 18 \%$ in the centrality class $20-40 \%$ for the $p_{\mathrm{T}}$ interval $0.5-0.7 \mathrm{GeV} / c$. It is observed to decrease with increasing $p_{\mathrm{T}}$ reaching $2 \%$ for $p_{\mathrm{T}}=$ $2 \mathrm{GeV} / c$, where the contribution of background electrons starts to become negligible. In pp collisions, the uncertainty arising from the photonic-electron subtraction is estimated to be about $3 \%$ with no $p_{\mathrm{T}}$ dependence. In addition, the dependence of the photonicelectron tagging efficiency on the spectral shape of the background sources is taken into account by recalculating the efficiency for different $\pi^{0}$ and $\eta p_{\mathrm{T}}$ spectra. The variation of the neutral-meson spectra is obtained by parameterising the measured spectra considering their systematic uncertainties. In particular, the measured yields at the lowest transverse 
momenta are shifted up by their systematic uncertainties and the yields at the highest transverse momenta are shifted down, and vice versa. The resulting systematic uncertainty on the spectra of electrons from semileptonic heavy-flavour hadron decays is $1 \%$ for $p_{\mathrm{T}} \leq 0.9 \mathrm{GeV} / c$ in $\mathrm{Pb}-\mathrm{Pb}$ collisions. In pp collisions, the systematic uncertainty is about $5 \%$ in the $p_{\mathrm{T}}$ interval $0.5-0.7 \mathrm{GeV} / c, 2 \%$ in $0.7-0.9 \mathrm{GeV} / c, 1 \%$ in $0.9-1.5 \mathrm{GeV} / c$ and negligible for higher $p_{\mathrm{T}}$. It is worth noting that replacing the previous approach to determine the photonic background via a cocktail calculation of the known sources [47] by an actual measurement of this background component resulted in a reduction of the related systematic uncertainties of the pp reference cross section by a factor of about 3 .

In order to further test the robustness of the photonic-electron tagging, the number of clusters required for electron candidates in the SPD has been released to a single hit in any of the two layers, increasing in this way the fraction of electrons coming from photon conversions in the detector material. In the pp analysis, a contribution to the systematic uncertainties of about $20 \%$ in the $p_{\mathrm{T}}$ interval $0.5-0.7 \mathrm{GeV} / c$ and $5 \%$ up to $p_{\mathrm{T}}=1.3 \mathrm{GeV} / c$ is assigned, while for higher $p_{\mathrm{T}}$ this uncertainty is estimated to be negligible. In the $\mathrm{Pb}-\mathrm{Pb}$ case the systematic uncertainty is $3 \%$ with no $p_{\mathrm{T}}$ and centrality dependence. This systematic uncertainty is significantly larger for the pp sample because of the specific detector configuration. Due to the lack of the SDD detector information at track reconstruction level, only a maximum of four hits in the ITS can be expected instead of the usual six. Therefore, this sample is potentially affected by a higher fraction of badly reconstructed tracks, particularly at the lowest transverse momenta. In addition to releasing the condition on the SPD layers, the systematic uncertainty in the pp case has been determined by comparing the measurement obtained from the analysis of a sub-set of events where all six ITS layers are used for the track reconstruction.

The subtraction of the background electron contribution from the $\mathrm{J} / \psi$ and $\mathrm{K}_{e 3}$ decays is affected by the uncertainty on the input distribution used for the cocktail calculation. This results in an uncertainty of $4 \%$ and $2 \%$ in the lowest $p_{\mathrm{T}}$ interval in pp and in $\mathrm{Pb}-\mathrm{Pb}$ collisions, respectively. While for pp collisions this contribution is negligible at higher $p_{\mathrm{T}}$, for $\mathrm{Pb}-\mathrm{Pb}$ collisions it decreases slowly with increasing $p_{\mathrm{T}}$, reaching a minimum of $1 \%$ at $p_{\mathrm{T}}=1.5 \mathrm{GeV} / c$ before increasing again to $4 \%$ at $p_{\mathrm{T}}=3 \mathrm{GeV} / c$ due to the growing contribution from $\mathrm{J} / \psi$ decays.

Events with a primary vertex reconstructed using charged-particle tracks are used. For the pp analysis, the resolution of the vertex position is affected by the absence of the $\mathrm{SDD}$ information and by the lower multiplicity of tracks compared to the $\mathrm{Pb}-\mathrm{Pb}$ case. The associated uncertainty of $3 \%$ is estimated by comparing the cross sections measured from events where the vertex was determined either with charged-particle tracks or with the SPD information only.

\section{Results}

\section{1 $p_{\mathrm{T}}$-differential invariant cross section in pp collisions}

The measurement presented in this paper for pp collisions updates the charge averaged $p_{\mathrm{T}^{-}}$ differential cross section published previously [47] in the range $p_{\mathrm{T}}<3.0 \mathrm{GeV} / c$. The new 


\begin{tabular}{|c|c|c|c|c|c|c|}
\hline Collision system & \multicolumn{2}{|c|}{$\mathrm{Pb}-\mathrm{Pb}(0-10 \%)$} & \multicolumn{2}{|c|}{$\mathrm{Pb}-\mathrm{Pb}(20-40 \%)$} & \multicolumn{2}{|c|}{ pp } \\
\hline$p_{\mathrm{T}}$ interval $(\mathrm{GeV} / c)$ & $0.5-0.7$ & $2-3$ & $0.5-0.7$ & $2-3$ & $0.5-0.7$ & $2-3$ \\
\hline Electron candidate selection & $6 \%$ & $3 \%$ & $6 \%$ & $3 \%$ & $4 \%$ & \\
\hline Photonic electron subtraction & $30 \%$ & $2 \%$ & $18 \%$ & $2 \%$ & $3 \%$ & \\
\hline$\pi^{0}$ and $\eta$ Weights & $1 \%$ & - & $1 \%$ & - & $5 \%$ & - \\
\hline $\mathrm{SPD}$ requirement & \multicolumn{2}{|c|}{$3 \%$} & \multicolumn{2}{|c|}{$3 \%$} & $20 \%$ & - \\
\hline Track matching & \multicolumn{2}{|c|}{$5 \%$} & \multicolumn{2}{|c|}{$5 \%$} & $4 \%$ & \\
\hline Magnet polarity & $2 \%$ & - & $2 \%$ & - & - & \\
\hline Rapidity range & $5 \%$ & - & $5 \%$ & - & $5 \%$ & - \\
\hline Event selection & \multicolumn{2}{|c|}{ - } & \multicolumn{2}{|c|}{ - } & $3 \%$ & \\
\hline Subtraction of $\mathrm{J} / \psi$ and $\mathrm{K}_{e 3}$ & $2 \%$ & $4 \%$ & $2 \%$ & $3 \%$ & $4 \%$ & - \\
\hline Total systematic uncertainty & $32 \%$ & $8 \%$ & $21 \%$ & $7 \%$ & $23 \%$ & $7 \%$ \\
\hline
\end{tabular}

Table 5. Contributions to the systematic uncertainties on the yield of electrons from semileptonic heavy-flavour hadron decays, quoted for the lowest and highest $p_{\mathrm{T}}$ interval, respectively.

$p_{\mathrm{T}}$-differential invariant cross section for electrons from semileptonic heavy-flavour hadron decays measured at mid-rapidity in pp collisions at $\sqrt{s}=2.76 \mathrm{TeV}$ is shown in Figure 1. Results from a previous publication [47] (open circles in Figure 1) are plotted together with the new results from the TPC-TOF analysis (filled circles in Figure 1) reported in the current paper. Applying the photonic tagging background subtraction method [45] allowed for a reduction of the systematic uncertainties of the pp reference cross section by a factor of about 3 compared to the previously published reference [47], which is consistent within uncertainties with the current measurement. The cross section from a pQCD calculation employing the Fixed-Order-Next-to-Leading-Log (FONLL) scheme [77] is compared with the data in Figure 1. The uncertainties of the FONLL calculations (red dashed area) reflect different choices for the charm and beauty quark masses, the factorization and renormalization scales as well as from the uncertainty on the set of parton distribution functions used in the pQCD calculation (CTEQ6.6 [78]). The result from the FONLL calculation is consistent with the measured production cross section of electrons from semileptonic heavy-flavour hadron decays. The measured cross section is close to the upper edge of the FONLL uncertainty band, as it was observed previously in pp collisions at the LHC [47, 59] and at RHIC, for $p_{\mathrm{T}}>1.5 \mathrm{GeV} / c$ [23, 24], as well as in $\mathrm{p} \overline{\mathrm{p}}$ collisions at the Tevatron [79].

\section{$4.2 \quad p_{\mathrm{T}}$-differential invariant yields in $\mathrm{Pb}-\mathrm{Pb}$ collisions}

The charge averaged $p_{\mathrm{T}}$-differential invariant yields of electrons and positrons from semileptonic heavy-flavour hadron decays measured in the range $0.5<p_{\mathrm{T}}<3 \mathrm{GeV} / c$ at mid rapidity in $0-10 \%$ (black circles) and $20-40 \%$ (red squares) central $\mathrm{Pb}-\mathrm{Pb}$ collisions at $\sqrt{s_{\mathrm{NN}}}=2.76 \mathrm{TeV}$ are depicted in Figure 2. 


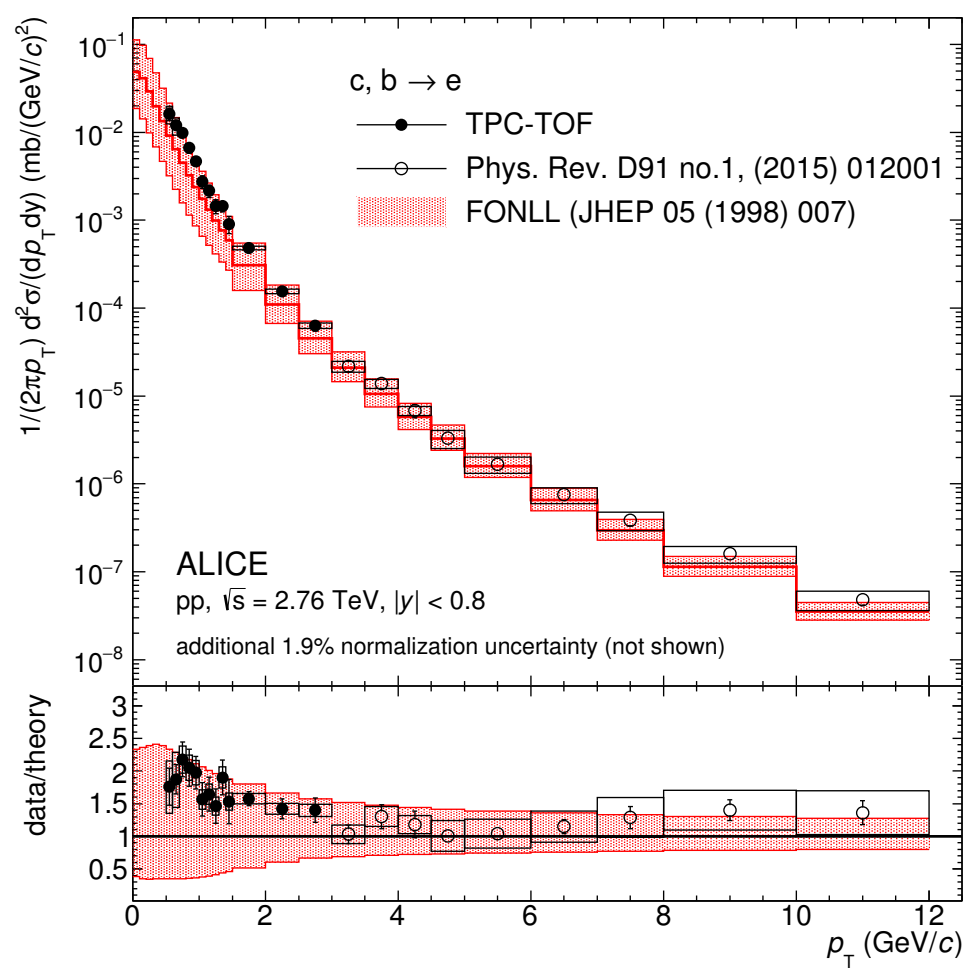

Figure 1. The $p_{\mathrm{T}}$-differential invariant production cross section for electrons from semileptonic heavy-flavour hadron decays measured at mid-rapidity in pp collisions at $\sqrt{s}=2.76 \mathrm{TeV}$ in comparison with FONLL pQCD calculations [77] (upper panel), and the ratio of the data to the FONLL calculation (lower panel). Statistical and systematic uncertainties are shown as vertical bars and boxes, respectively.

\subsection{Nuclear modification factor $R_{\mathrm{AA}}$}

Figure 3 shows the nuclear modification factor of electrons from semileptonic heavy-flavour hadron decays at mid-rapidity as a function of $p_{\mathrm{T}}$ in $\mathrm{Pb}-\mathrm{Pb}$ collisions at $\sqrt{s_{\mathrm{NN}}}=2.76 \mathrm{TeV}$ for the $0-10 \%$ (left panel) and 20-40\% (right panel) centrality classes. The low- $p_{\mathrm{T}}$ data from the current analysis (filled symbols) are shown together with the previously published [42] high- $p_{\mathrm{T}} R_{\mathrm{AA}}$ (open symbols). The $20-30 \%$ and $30-40 \%$ centrality intervals from [42], in which electrons were identified using the specific energy loss in the TPC and electromagnetic showers reconstructed in the electromagnetic calorimeter (EMCal) of ALICE, have been combined. Statistical and systematic uncertainties of the $p_{\mathrm{T}}$-differential yields and cross sections in $\mathrm{Pb}-\mathrm{Pb}$ and $\mathrm{pp}$ collisions, respectively, are propagated as uncorrelated uncertainties. The $1.9 \%$ normalization uncertainty on the pp measurement is included in the systematic uncertainties of the invariant cross section, and summed in quadrature with the other systematic uncertainties. The uncertainties of the average nuclear overlap function $\left\langle T_{\mathrm{AA}}\right\rangle$ in the $0-10 \%$ and $20-40 \%$ centrality classes are represented by the boxes at $R_{\mathrm{AA}}=1$. For $p_{\mathrm{T}}>3 \mathrm{GeV} / c$ the yield of electrons from heavy-flavour hadron decays is suppressed strongly which was interpreted as due to partonic energy loss in the QGP produced in $\mathrm{Pb}-\mathrm{Pb}$ collisions [42]. The current measurement provides an extension of the $p_{\mathrm{T}}$ coverage 


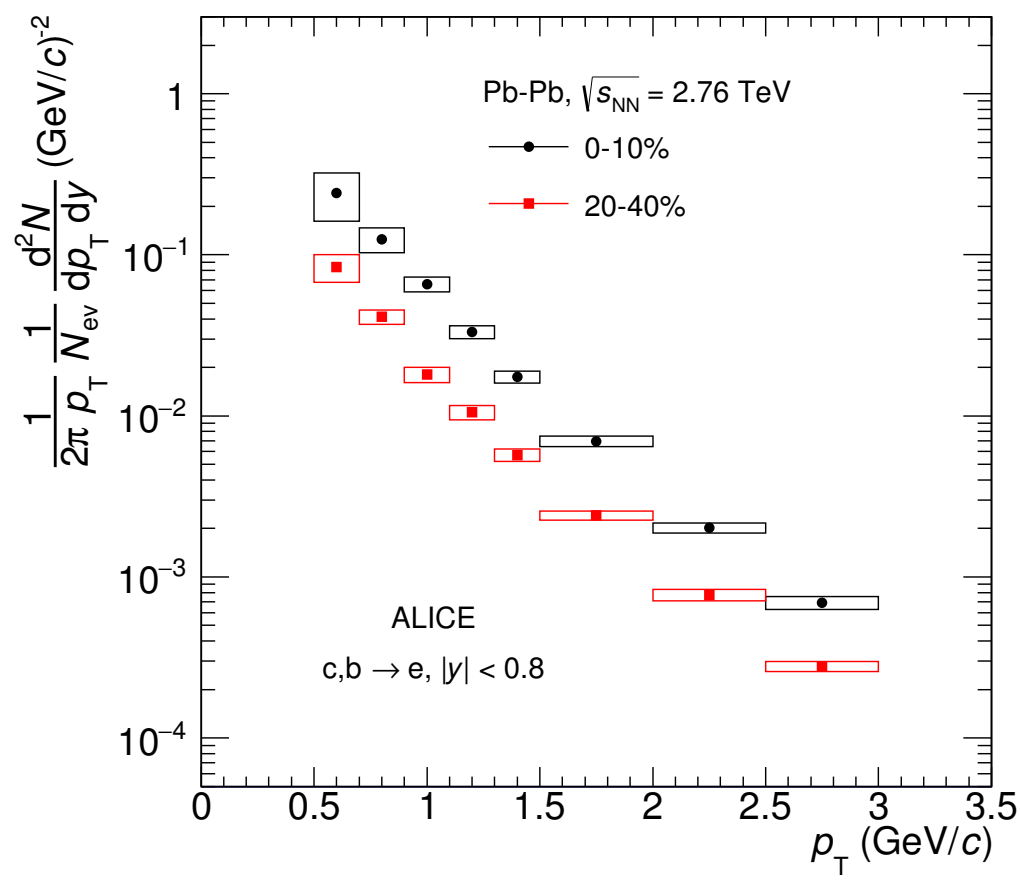

Figure 2. The $p_{\mathrm{T}}$-differential invariant yields of electrons from semileptonic heavy-flavour hadron decays measured at mid-rapidity in $0-10 \%$ and $20-40 \%$ central $\mathrm{Pb}-\mathrm{Pb}$ collisions at $\sqrt{s_{\mathrm{NN}}}=$ $2.76 \mathrm{TeV}$. Statistical uncertainties are smaller than the symbol size and the systematic uncertainties are shown as boxes.

to lower values, i.e. from $p_{\mathrm{T}}=3 \mathrm{GeV} / c$ down to $0.5 \mathrm{GeV} / c$. In this region, the suppression of the yield of electrons from heavy-flavour hadron decays is expected to decrease with decreasing $p_{\mathrm{T}}$ as a consequence of the scaling of the total heavy-flavour yield with the number of binary collisions in $\mathrm{Pb}-\mathrm{Pb}$ collisions. This scaling, however can be broken due to the nuclear modification of the parton distribution functions in $\mathrm{Pb}$-nuclei, leading to $p_{\mathrm{T}}$-integrated $R_{\mathrm{AA}}$ of less than one. Moreover, further modifications of the $p_{\mathrm{T}}$ distribution due to the radial flow can also play a role in this region. The observed $R_{\mathrm{AA}}$ in figure 3 is consistent with the expectation of an increasing $R_{\mathrm{AA}}$ with decreasing $p_{\mathrm{T}}$, reaching values close to unity within uncertainties. However the current uncertainties are still too large to quantify the different effects. Within the current statistical and systematic uncertainties, no significant centrality dependence is observed in the $p_{\mathrm{T}}$-region below $3 \mathrm{GeV} / c$.

\section{Comparison with model calculations}

In Figure 4 results from model calculations including charm and beauty quark interactions with a QGP medium [80-85] are compared with the measured $R_{\mathrm{AA}}$ of electrons from semileptonic heavy-flavour hadron decays for the $10 \%$ most central $\mathrm{Pb}-\mathrm{Pb}$ collisions. The calculations differ in the modelling of the initial conditions, the medium properties, the dynamics of the medium evolution, the interactions of charm and beauty quarks with the QGP, and in the implementation of hadronisation and hadronic interactions in the late stages of the heavy-ion collision. Furthermore, there are differences in the initial 


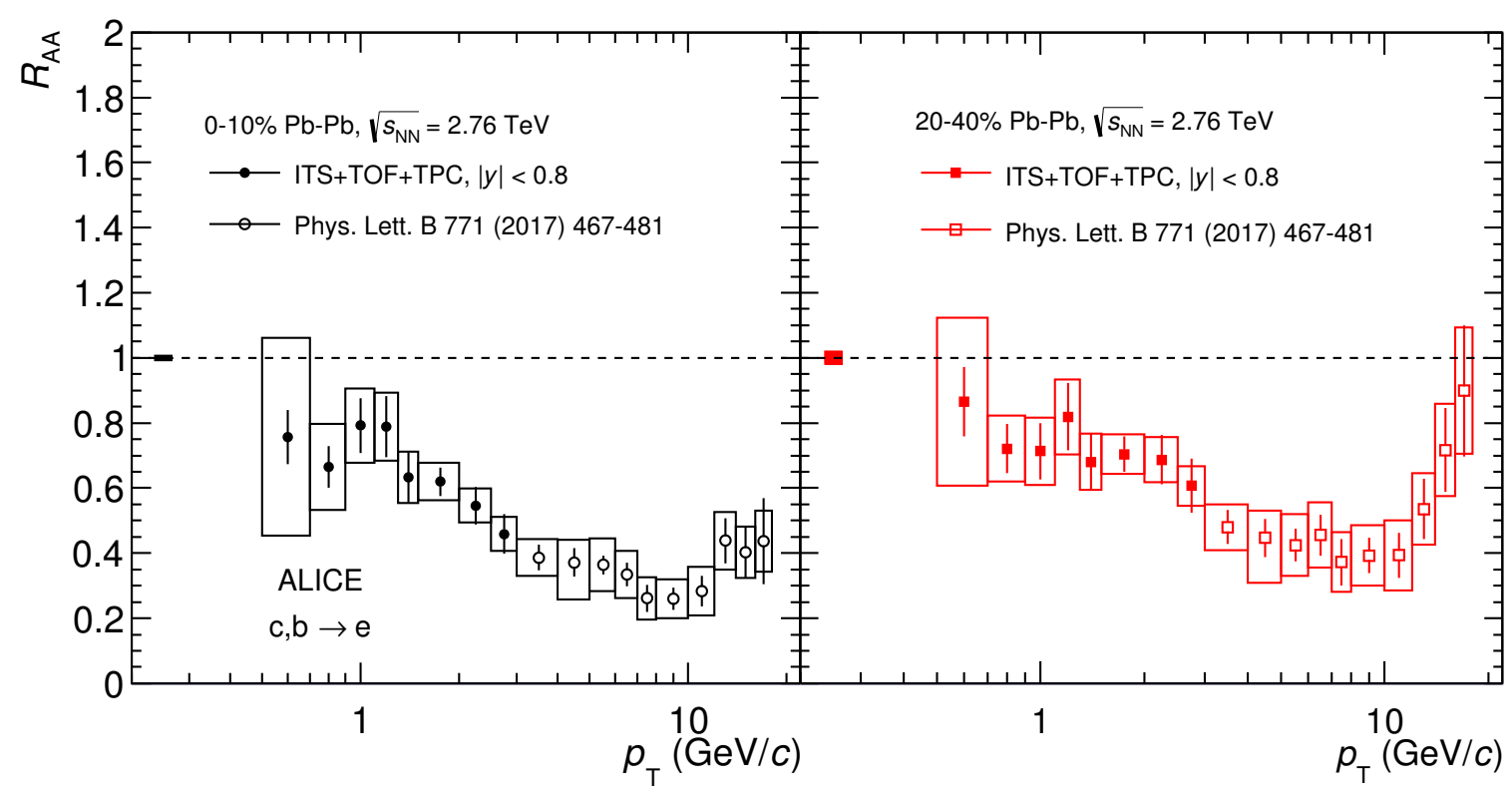

Figure 3. Nuclear modification factor $R_{\mathrm{AA}}$ for electrons from semileptonic heavy-flavour hadron decays at mid-rapidity as a function of $p_{\mathrm{T}}$ in 0-10\% (left panel) and 20-40\% central (right panel) $\mathrm{Pb}-$ $\mathrm{Pb}$ collisions at $\sqrt{s_{\mathrm{NN}}}=2.76 \mathrm{TeV}$. Error bars (open boxes) represent the statistical (systematic) uncertainties. The normalization uncertainties are represented by the boxes at $R_{\mathrm{AA}}=1$. The previously published results from [42] have been updated using a new glauber model calculation [60].

$p_{\mathrm{T}}$-differential heavy-quark production cross section in nucleon-nucleon collisions used as input. Qualitatively, most models provide a good description of the heavy-flavour $R_{\mathrm{AA}}$ measured in the most central $\mathrm{Pb}-\mathrm{Pb}$ collisions as already observed for $\mathrm{D}$ mesons [42].

The measurement presented in this paper shows for the first time electrons from heavyflavour hadron decays in the $p_{\mathrm{T}}$ interval below $1 \mathrm{GeV} / c$, where decays of heavy-flavour hadrons down to zero $p_{\mathrm{T}}$ contribute. In this region, the nuclear modifications of the PDFs can play a significant role [39-42]. This is addressed in Figure 5, which compares the measured nuclear modification factor with TAMU, POWLANG and MC@sHQ+EPOS2 model calculations with and without the inclusion of the EPS09 shadowing parameterisations [34]. The depletion of the parton densities at low $x$, resulting in a reduced heavy-flavour production cross section per nucleon-nucleon pair in $\mathrm{Pb}-\mathrm{Pb}$ collisions with respect to bare nucleon-nucleon collisions, leads to a reduction of $R_{\mathrm{AA}}$ of electrons from heavy-flavour hadron decays at low $p_{\mathrm{T}}$. Data are better described when the nuclear PDFs are included in the theoretical calculation in both centrality intervals. However, the experimental uncertainties are still too large to provide quantitative constraints on the nuclear shadowing contribution. A similar conclusion arises from measurements of D-meson production in $\mathrm{Pb}-\mathrm{Pb}$ collisions [43].

\section{Conclusions}

The production of electrons from semileptonic decays of heavy-flavour hadrons has been measured at mid-rapidity $(|y|<0.8)$ in the $p_{\mathrm{T}}$ interval $0.5-3 \mathrm{GeV} / c$ in pp collisions and 


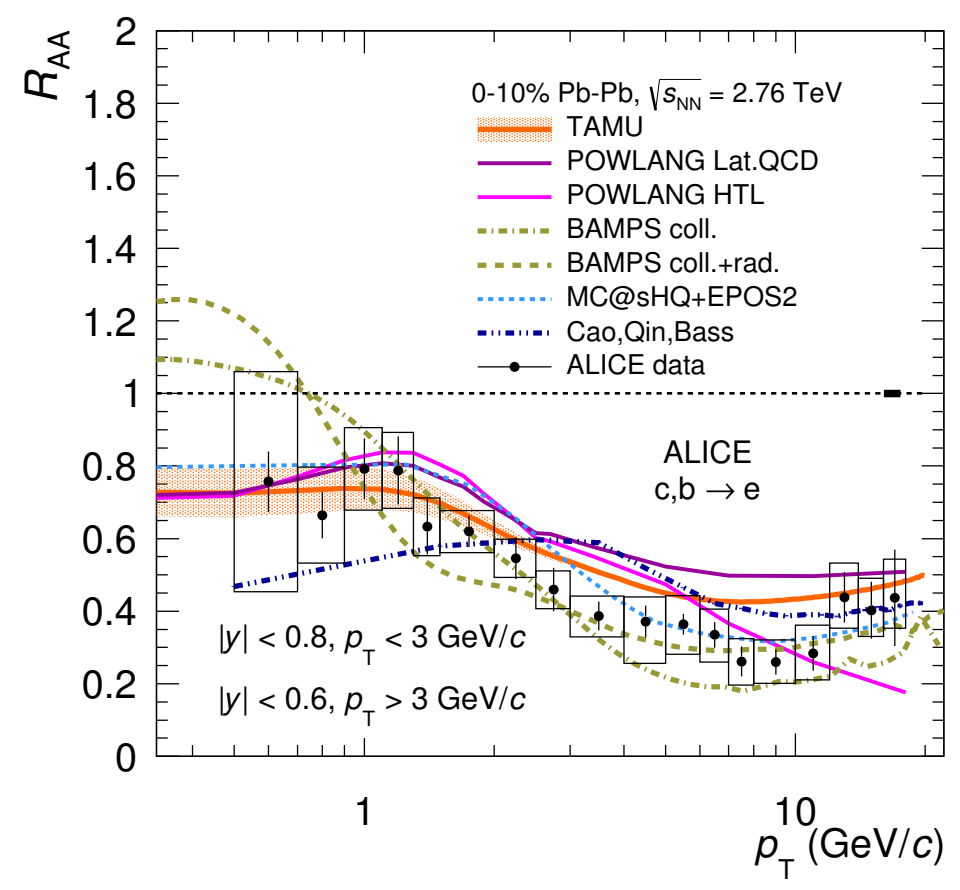

Figure 4. $R_{\mathrm{AA}}$ of electrons from semileptonic heavy-flavour hadron decays at mid-rapidity as a function of $p_{\mathrm{T}}$ in $0-10 \% \mathrm{~Pb}-\mathrm{Pb}$ collisions at $\sqrt{s_{\mathrm{NN}}}=2.76 \mathrm{TeV}$ compared to model calculations $[80-85]$.
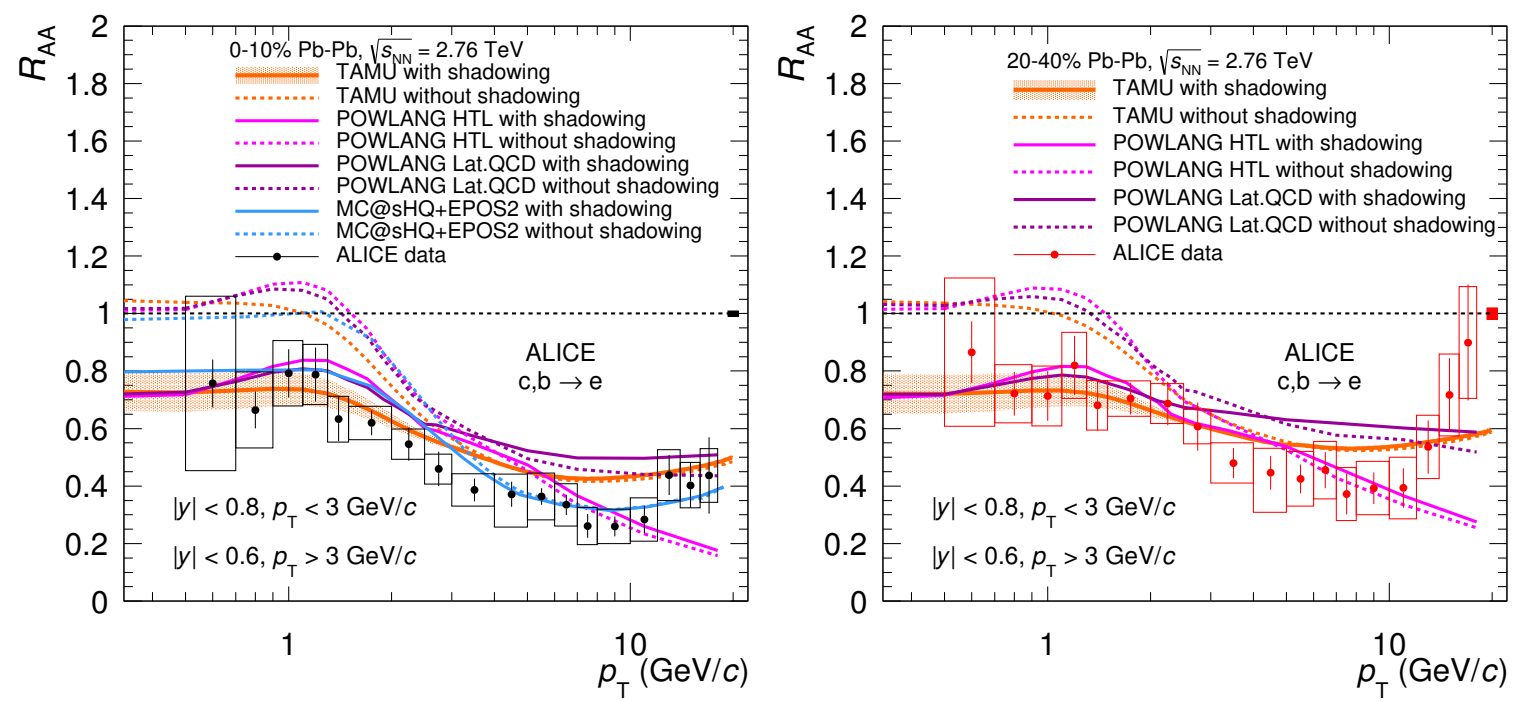

Figure 5. $R_{\mathrm{AA}}$ of electrons from semileptonic heavy-flavour hadron decays at mid-rapidity as a function of $p_{\mathrm{T}}$ in $0-10 \%$ (left) and $20-40 \%$ central (right) $\mathrm{Pb}-\mathrm{Pb}$ collisions at $\sqrt{s_{\mathrm{NN}}}=2.76 \mathrm{TeV}$ compared to model calculations [82] with and without EPS09 shadowing parameterisations [34]. 
in $0-10 \%$ and $20-40 \%$ central $\mathrm{Pb}-\mathrm{Pb}$ collisions at a centre-of-mass energy of $2.76 \mathrm{TeV}$ per nucleon pair. The dominant background from photonic electron sources has been measured and subtracted via the photonic-electron tagging technique for the first time in pp and $\mathrm{Pb}-\mathrm{Pb}$ collisions at the same energy. The systematic uncertainties have been substantially reduced (up to a factor 3 ), and the $p_{\mathrm{T}}$ coverage has been extended to lower values with respect to previously published ALICE measurements.

The measured nuclear modification factor $R_{\mathrm{AA}}$ of electrons from semileptonic heavyflavour hadron decays confirms the strong suppression of high- $p_{\mathrm{T}}$ heavy-flavour hadrons in central $\mathrm{Pb}-\mathrm{Pb}$ collisions with respect to the binary-collision scaled pp reference, consistent with previous observations in various heavy-flavour channels. With decreasing $p_{\mathrm{T}}, R_{\mathrm{AA}}$ grows approaching values close to unity, as expected from the hypothesis of the binarycollision scaling for the total heavy-quark yield. However, this kinematic region is sensitive to the effects of nuclear shadowing: the depletion of parton densities in nuclei at low Bjorken $x$ values can reduce the heavy-quark production cross section per binary collision in $\mathrm{Pb}-$ $\mathrm{Pb}$ with respect to the pp case. This initial-state effect is studied in $\mathrm{p}-\mathrm{Pb}$ collisions [45]. However, the present uncertainties on the $R_{\mathrm{pPb}}$ measurement do not allow quantitative conclusions on the modification of the PDF in nuclei in the low $p_{\mathrm{T}}$ region. With the improved precision of the results presented here, the $\mathrm{Pb}-\mathrm{Pb}$ data exhibit their sensitivity to the modification of the PDF in nuclei, like nuclear shadowing, at low $p_{\mathrm{T}}$. The measured $R_{\mathrm{AA}}$ is in better agreement with TAMU and POWLANG model calculations when the nuclear modification of the PDF is included.

\section{Acknowledgments}

The ALICE Collaboration would like to thank all its engineers and technicians for their invaluable contributions to the construction of the experiment and the CERN accelerator teams for the outstanding performance of the LHC complex. The ALICE Collaboration gratefully acknowledges the resources and support provided by all Grid centres and the Worldwide LHC Computing Grid (WLCG) collaboration. The ALICE Collaboration acknowledges the following funding agencies for their support in building and running the ALICE detector: A. I. Alikhanyan National Science Laboratory (Yerevan Physics Institute) Foundation (ANSL), State Committee of Science and World Federation of Scientists (WFS), Armenia; Austrian Academy of Sciences and Nationalstiftung für Forschung, Technologie und Entwicklung, Austria; Ministry of Communications and High Technologies, National Nuclear Research Center, Azerbaijan; Conselho Nacional de Desenvolvimento Científico e Tecnológico (CNPq), Universidade Federal do Rio Grande do Sul (UFRGS), Financiadora de Estudos e Projetos (Finep) and Fundação de Amparo à Pesquisa do Estado de São Paulo (FAPESP), Brazil; Ministry of Science \& Technology of China (MSTC), National Natural Science Foundation of China (NSFC) and Ministry of Education of China (MOEC), China; Ministry of Science and Education, Croatia; Ministry of Education, Youth and Sports of the Czech Republic, Czech Republic; The Danish Council for Independent Research - Natural Sciences, the Carlsberg Foundation and Danish National Research Foundation (DNRF), Denmark; Helsinki Institute of Physics (HIP), Finland; Commissariat 
à l'Energie Atomique (CEA) and Institut National de Physique Nucléaire et de Physique des Particules (IN2P3) and Centre National de la Recherche Scientifique (CNRS), France; Bundesministerium für Bildung, Wissenschaft, Forschung und Technologie (BMBF) and GSI Helmholtzzentrum für Schwerionenforschung GmbH, Germany; General Secretariat for Research and Technology, Ministry of Education, Research and Religions, Greece; National Research, Development and Innovation Office, Hungary; Department of Atomic Energy Government of India (DAE), Department of Science and Technology, Government of India (DST), University Grants Commission, Government of India (UGC) and Council of Scientific and Industrial Research (CSIR), India; Indonesian Institute of Science, Indonesia; Centro Fermi - Museo Storico della Fisica e Centro Studi e Ricerche Enrico Fermi and Istituto Nazionale di Fisica Nucleare (INFN), Italy; Institute for Innovative Science and Technology, Nagasaki Institute of Applied Science (IIST), Japan Society for the Promotion of Science (JSPS) KAKENHI and Japanese Ministry of Education, Culture, Sports, Science and Technology (MEXT), Japan; Consejo Nacional de Ciencia (CONACYT) y Tecnología, through Fondo de Cooperación Internacional en Ciencia y Tecnología (FONCICYT) and Dirección General de Asuntos del Personal Academico (DGAPA), Mexico; Nederlandse Organisatie voor Wetenschappelijk Onderzoek (NWO), Netherlands; The Research Council of Norway, Norway; Commission on Science and Technology for Sustainable Development in the South (COMSATS), Pakistan; Pontificia Universidad Católica del Perú, Peru; Ministry of Science and Higher Education and National Science Centre, Poland; Korea Institute of Science and Technology Information and National Research Foundation of Korea (NRF), Republic of Korea; Ministry of Education and Scientific Research, Institute of Atomic Physics and Romanian National Agency for Science, Technology and Innovation, Romania; Joint Institute for Nuclear Research (JINR), Ministry of Education and Science of the Russian Federation and National Research Centre Kurchatov Institute, Russia; Ministry of Education, Science, Research and Sport of the Slovak Republic, Slovakia; National Research Foundation of South Africa, South Africa; Centro de Aplicaciones Tecnológicas y Desarrollo Nuclear (CEADEN), Cubaenergía, Cuba and Centro de Investigaciones Energéticas, Medioambientales y Tecnológicas (CIEMAT), Spain; Swedish Research Council (VR) and Knut \& Alice Wallenberg Foundation (KAW), Sweden; European Organization for Nuclear Research, Switzerland; National Science and Technology Development Agency (NSDTA), Suranaree University of Technology (SUT) and Office of the Higher Education Commission under NRU project of Thailand, Thailand; Turkish Atomic Energy Agency (TAEK), Turkey; National Academy of Sciences of Ukraine, Ukraine; Science and Technology Facilities Council (STFC), United Kingdom; National Science Foundation of the United States of America (NSF) and United States Department of Energy, Office of Nuclear Physics (DOE NP), United States of America.

Open Access. This article is distributed under the terms of the Creative Commons Attribution License (CC-BY 4.0), which permits any use, distribution and reproduction in any medium, provided the original author(s) and source are credited. 


\section{References}

[1] BRAHMS collaboration, I. Arsene et al., Quark gluon plasma and color glass condensate at RHIC? The Perspective from the BRAHMS experiment, Nucl. Phys. A 757 (2005) 1 [nucl-ex/0410020] [INSPIRE].

[2] PHENIX collaboration, K. Adcox et al., Formation of dense partonic matter in relativistic nucleus-nucleus collisions at RHIC: Experimental evaluation by the PHENIX collaboration, Nucl. Phys. A 757 (2005) 184 [nucl-ex/0410003] [InSPIRE].

[3] B.B. Back et al., The PHOBOS perspective on discoveries at RHIC, Nucl. Phys. A 757 (2005) 28 [nucl-ex/0410022] [INSPIRE].

[4] STAR collaboration, J. Adams et al., Experimental and theoretical challenges in the search for the quark gluon plasma: The STAR Collaboration's critical assessment of the evidence from RHIC collisions, Nucl. Phys. A 757 (2005) 102 [nucl-ex/0501009] [InSPIRE].

[5] ALICE collaboration, Elliptic flow of charged particles in $\mathrm{Pb}-\mathrm{Pb}$ collisions at 2.76 TeV, Phys. Rev. Lett. 105 (2010) 252302 [arXiv:1011.3914] [INSPIRE].

[6] ALICE collaboration, Suppression of Charged Particle Production at Large Transverse Momentum in Central Pb-Pb Collisions at $\sqrt{s_{\mathrm{NN}}}=2.76$ TeV, Phys. Lett. B 696 (2011) 30 [arXiv: 1012.1004] [INSPIRE].

[7] F. Karsch, Lattice simulations of the thermodynamics of strongly interacting elementary particles and the exploration of new phases of matter in relativistic heavy ion collisions, J. Phys. Conf. Ser. 46 (2006) 122 [hep-lat/0608003] [InSPIRE].

[8] Wuppertal-Budapest collaboration, S. Borsányi et al., Is there still any $T_{c}$ mystery in lattice QCD? Results with physical masses in the continuum limit III, JHEP 09 (2010) 073 [arXiv: 1005.3508] [INSPIRE].

[9] Wuppertal-Budapest collaboration, S. Borsányi et al., The QCD equation of state with dynamical quarks, JHEP 11 (2010) 077 [arXiv: 1007.2580] [INSPIRE].

[10] A. Bazavov et al., The chiral and deconfinement aspects of the QCD transition, Phys. Rev. D 85 (2012) 054503 [arXiv: 1111.1710] [INSPIRE].

[11] P. Petreczky, Review of recent highlights in lattice calculations at finite temperature and finite density, PoS (Confinement X) 028 [arXiv: 1301.6188] [INSPIRE].

[12] F.-M. Liu and S.-X. Liu, quark-gluon plasma formation time and direct photons from heavy ion collisions, Phys. Rev. C 89 (2014) 034906 [arXiv: 1212.6587] [INSPIRE].

[13] R. Averbeck, Heavy-flavor production in heavy-ion collisions and implications for the properties of hot QCD matter, Prog. Part. Nucl. Phys. 70 (2013) 159 [arXiv:1505.03828] [INSPIRE].

[14] F. Prino and R. Rapp, Open Heavy Flavor in QCD Matter and in Nuclear Collisions, J. Phys. G 43 (2016) 093002 [arXiv: 1603.00529] [INSPIRE].

[15] P. Braun-Munzinger, Quarkonium production in ultra-relativistic nuclear collisions: Suppression versus enhancement, J. Phys. G 34 (2007) S471 [nucl-th/0701093] [INSPIRE].

[16] R.J. Glauber and G. Matthiae, High-energy scattering of protons by nuclei, Nucl. Phys. B 21 (1970) 135 [INSPIRE].

[17] M.L. Miller, K. Reygers, S.J. Sanders and P. Steinberg, Glauber modeling in high energy nuclear collisions, Ann. Rev. Nucl. Part. Sci. 57 (2007) 205 [nucl-ex/0701025] [INSPIRE]. 
[18] M. Gyulassy and M. Plumer, Jet Quenching in Dense Matter, Phys. Lett. B 243 (1990) 432 [INSPIRE].

[19] R. Baier, Y.L. Dokshitzer, A.H. Mueller, S. Peigne and D. Schiff, Radiative energy loss and $p_{T}$ broadening of high-energy partons in nuclei, Nucl. Phys. B 484 (1997) 265 [hep-ph/9608322] [INSPIRE].

[20] M.H. Thoma and M. Gyulassy, Quark Damping and Energy Loss in the High Temperature QCD, Nucl. Phys. B 351 (1991) 491 [INSPIRE].

[21] E. Braaten and M.H. Thoma, Energy loss of a heavy fermion in a hot plasma, Phys. Rev. D 44 (1991) 1298 [INSPIRE].

[22] E. Braaten and M.H. Thoma, Energy loss of a heavy quark in the quark-gluon plasma, Phys. Rev. D 44 (1991) R2625 [inSPIRE]

[23] STAR collaboration, B.I. Abelev et al., Transverse momentum and centrality dependence of high-p $p_{T}$ non-photonic electron suppression in Au+Au collisions at $\sqrt{s_{\mathrm{NN}}}=200 \mathrm{GeV}$, Phys. Rev. Lett. 98 (2007) 192301 [Erratum ibid. 106 (2011) 159902] [nucl-ex/0607012] [INSPIRE].

[24] PHENIX collaboration, A. Adare et al., Heavy Quark Production in $p+p$ and Energy Loss and Flow of Heavy Quarks in Au+Au Collisions at $\sqrt{s_{\mathrm{NN}}}=200 \mathrm{GeV}$, Phys. Rev. C 84 (2011) 044905 [arXiv: 1005.1627] [INSPIRE].

[25] PHENIX collaboration, S.S. Adler et al., Nuclear modification of electron spectra and implications for heavy quark energy loss in Au+Au collisions at $\sqrt{s_{\mathrm{NN}}}=200 \mathrm{GeV}$, Phys. Rev. Lett. 96 (2006) 032301 [nucl-ex/0510047] [INSPIRE].

[26] PHENIX collaboration, A. Adare et al., System-size dependence of open-heavy-flavor production in nucleus-nucleus collisions at $\sqrt{s_{\mathrm{NN}}}=200 \mathrm{GeV}$, Phys. Rev. C 90 (2014) 034903 [arXiv: 1310.8286] [INSPIRE].

[27] STAR collaboration, L. Adamczyk et al., Observation of $D^{0}$ Meson Nuclear Modifications in Au + Au Collisions at $\sqrt{s_{\mathrm{NN}}}=200 \mathrm{GeV}$, Phys. Rev. Lett. 113 (2014) 142301 [arXiv: 1404.6185] [INSPIRE].

[28] ALICE collaboration, Elliptic flow of electrons from heavy-flavour hadron decays at mid-rapidity in $\mathrm{Pb}-\mathrm{Pb}$ collisions at $\sqrt{s_{\mathrm{NN}}}=2.76 \mathrm{TeV}$, JHEP 09 (2016) 028 [arXiv: 1606.00321] [INSPIRE].

[29] ALICE collaboration, $D$ meson elliptic flow in non-central $\mathrm{Pb}-\mathrm{Pb}$ collisions at $\sqrt{s_{\mathrm{NN}}}=2.76 \mathrm{TeV}$, Phys. Rev. Lett. 111 (2013) 102301 [arXiv:1305.2707] [INSPIRE].

[30] H. van Hees, V. Greco and R. Rapp, Heavy-quark probes of the quark-gluon plasma at RHIC, Phys. Rev. C 73 (2006) 034913 [nucl-th/0508055] [inSPIRE].

[31] V. Greco, C.M. Ko and R. Rapp, Quark coalescence for charmed mesons in ultrarelativistic heavy ion collisions, Phys. Lett. B 595 (2004) 202 [nucl-th/0312100] [INSPIRE].

[32] A. Andronic, P. Braun-Munzinger, K. Redlich and J. Stachel, Statistical hadronization of charm in heavy ion collisions at SPS, RHIC and LHC, Phys. Lett. B 571 (2003) 36 [nucl-th/0303036] [INSPIRE].

[33] A. Andronic et al., Heavy-flavour and quarkonium production in the LHC era: from proton-proton to heavy-ion collisions, Eur. Phys. J. C 76 (2016) 107 [arXiv:1506.03981] [INSPIRE].

[34] K.J. Eskola, H. Paukkunen and C.A. Salgado, EPSO9: A New Generation of NLO and LO Nuclear Parton Distribution Functions, JHEP 04 (2009) 065 [arXiv: 0902.4154] [INSPIRE]. 
[35] B.Z. Kopeliovich, J. Nemchik, A. Schafer and A.V. Tarasov, Cronin effect in hadron production off nuclei, Phys. Rev. Lett. 88 (2002) 232303 [hep-ph/0201010] [INSPIRE].

[36] CMS collaboration, Nuclear modification factor of $D^{0}$ mesons in $\mathrm{Pb}-\mathrm{Pb}$ collisions at $\sqrt{s_{\mathrm{NN}}}=5.02 \mathrm{TeV}$, Phys. Lett. B $782(2018) 474$ [arXiv:1708.04962] [INSPIRE].

[37] CMS collaboration, Measurement of the $B^{ \pm}$Meson Nuclear Modification Factor in $\mathrm{Pb}-\mathrm{Pb}$ Collisions at $\sqrt{s_{\mathrm{NN}}}=5.02 \mathrm{TeV}$, Phys. Rev. Lett. 119 (2017) 152301 [arXiv:1705.04727] [INSPIRE].

[38] ALICE collaboration, Measurement of electrons from beauty-hadron decays in $p$ - $P b$ collisions at $\sqrt{s_{\mathrm{NN}}}=5.02 \mathrm{TeV}$ and $\mathrm{Pb}-\mathrm{Pb}$ collisions at $\sqrt{s_{\mathrm{NN}}}=2.76 \mathrm{TeV}$, JHEP 07 (2017) 052 [arXiv: 1609.03898] [INSPIRE].

[39] ALICE collaboration, Suppression of high transverse momentum D mesons in central $P b-P b$ collisions at $\sqrt{s_{\mathrm{NN}}}=2.76 \mathrm{TeV}$, JHEP 09 (2012) 112 [arXiv: 1203.2160] [INSPIRE].

[40] ALICE collaboration, Centrality dependence of high- $p_{T} D$ meson suppression in $\mathrm{Pb}-\mathrm{Pb}$ collisions at $\sqrt{s_{\mathrm{NN}}}=2.76 \mathrm{TeV}$, JHEP 11 (2015) 205 [arXiv: 1506.06604] [INSPIRE].

[41] ALICE collaboration, Production of muons from heavy flavour decays at forward rapidity in $p p$ and $P b-P b$ collisions at $\sqrt{s_{\mathrm{NN}}}=2.76 \mathrm{TeV}$, Phys. Rev. Lett. 109 (2012) 112301 [arXiv: 1205.6443] [INSPIRE].

[42] ALICE collaboration, Measurement of the production of high- $p_{\mathrm{T}}$ electrons from heavy-flavour hadron decays in $\mathrm{Pb}-\mathrm{Pb}$ collisions at $\sqrt{s_{\mathrm{NN}}}=2.76 \mathrm{TeV}$, Phys. Lett. B 771 (2017) 467 [arXiv: 1609.07104] [INSPIRE].

[43] ALICE collaboration, Transverse momentum dependence of D-meson production in $\mathrm{Pb}-\mathrm{Pb}$ collisions at $\sqrt{s_{\mathrm{NN}}}=2.76 \mathrm{TeV}$, JHEP 03 (2016) 081 [arXiv:1509.06888] [INSPIRE].

[44] CMS collaboration, Study of B Meson Production in $p+P b$ Collisions at $\sqrt{s_{\mathrm{NN}}}=5.02 \mathrm{TeV}$ Using Exclusive Hadronic Decays, Phys. Rev. Lett. 116 (2016) 032301 [arXiv:1508. 06678] [INSPIRE].

[45] ALICE collaboration, Measurement of electrons from heavy-flavour hadron decays in $p$ - $P b$ collisions at $\sqrt{s_{\mathrm{NN}}}=5.02 \mathrm{TeV}$, Phys. Lett. B 754 (2016) 81 [arXiv:1509.07491] [INSPIRE].

[46] ALICE collaboration, Measurement of prompt D-meson production in $p-P b$ collisions at $\sqrt{s_{\mathrm{NN}}}=5.02 \mathrm{TeV}$, Phys. Rev. Lett. 113 (2014) 232301 [arXiv:1405.3452] [INSPIRE].

[47] ALICE collaboration, Measurement of electrons from semileptonic heavy-flavor hadron decays in pp collisions at $\sqrt{s}=2.76 \mathrm{TeV}$, Phys. Rev. D 91 (2015) 012001 [arXiv:1405.4117] [INSPIRE].

[48] ATLAS collaboration, Measurement of the suppression and elliptic anisotropy of heavy flavor muons in $\mathrm{Pb}+\mathrm{Pb}$ collisions at $\sqrt{s_{\mathrm{NN}}}=2.76 \mathrm{TeV}$ with the ATLAS detector, ATLAS-CONF-2015-053 (2015).

[49] ALICE collaboration, The ALICE experiment at the CERN LHC, 2008 JINST 3 S08002 [INSPIRE].

[50] ALICE collaboration, Performance of the ALICE Experiment at the CERN LHC, Int. J. Mod. Phys. A 29 (2014) 1430044 [arXiv:1402 .4476] [InSPIRE].

[51] ALICE collaboration, Alignment of the ALICE Inner Tracking System with cosmic-ray tracks, 2010 JINST 5 P03003 [arXiv: 1001.0502] [INSPIRE].

[52] J. Alme et al., The ALICE TPC, a large 3-dimensional tracking device with fast readout for ultra-high multiplicity events, Nucl. Instrum. Meth. A 622 (2010) 316 [arXiv:1001.1950] [INSPIRE]. 
[53] ALICE collaboration, Performance of the ALICE Time-Of-Flight detector at the LHC, Eur. Phys. J. Plus. 128 (2013) 44.

[54] ALICE collaboration, Performance of the ALICE VZERO system, 2013 JINST 8 P10016 [arXiv: 1306.3130] [INSPIRE].

[55] ALICE collaboration, Centrality determination of $\mathrm{Pb}-\mathrm{Pb}$ collisions at $\sqrt{s_{\mathrm{NN}}}=2.76 \mathrm{TeV}$ with ALICE, Phys. Rev. C 88 (2013) 044909 [arXiv: 1301.4361] [InSPIRE].

[56] C. Loizides, J. Nagle and P. Steinberg, Improved version of the PHOBOS Glauber Monte Carlo, SoftwareX 1-2 (2015) 13 [arXiv:1408.2549] [inSPIRE].

[57] B. Alver, M. Baker, C. Loizides and P. Steinberg, The PHOBOS Glauber Monte Carlo, arXiv:0805.4411 [INSPIRE].

[58] ALICE collaboration, Performance of a forward hadron calorimeter for the alice experiment, IEEE Nucl. Sci. Symp. Conf. Rec. 1 (1998) 8.

[59] ALICE collaboration, Measurement of electrons from semileptonic heavy-flavour hadron decays in pp collisions at $\sqrt{s}=7$ TeV, Phys. Rev. D 86 (2012) 112007 [arXiv:1205.5423] [INSPIRE].

[60] ALICE collaboration, Centrality determination in heavy ion collisions, ALICE-PUBLIC-2018-011 (2018).

[61] H. Bethe, Zur Theorie des Durchgangs schneller Korpuskularstrahlen durch Materie, Annalen Phys. 397 (1930) 325.

[62] STAR collaboration, L. Adamczyk et al., Elliptic flow of electrons from heavy-flavor hadron decays in $A u+A u$ collisions at $\sqrt{s_{\mathrm{NN}}}=200,62.4$ and $39 \mathrm{GeV}$, Phys. Rev. C 95 (2017) 034907 [arXiv: 1405.6348] [INSPIRE].

[63] M. Gyulassy and X.-N. Wang, HIJING 1.0: A Monte Carlo program for parton and particle production in high-energy hadronic and nuclear collisions, Comput. Phys. Commun. $\mathbf{8 3}$ (1994) 307 [nucl-th/9502021] [INSPIRE].

[64] T. Sjöstrand, S. Mrenna and P.Z. Skands, PYTHIA 6.4 Physics and Manual, JHEP 05 (2006) 026 [hep-ph/0603175] [INSPIRE].

[65] R. Brun, F. Carminati and S. Giani, GEANT Detector Description and Simulation Tool, CERN-W5013 (2008) [INSPIRE].

[66] ALICE collaboration, Neutral pion production at midrapidity in pp and $\mathrm{Pb}-\mathrm{Pb}$ collisions at $\sqrt{s_{\mathrm{NN}}}=2.76 \mathrm{TeV}$, Eur. Phys. J. C 74 (2014) 3108 [arXiv: 1405.3794] [INSPIRE].

[67] ALICE collaboration, Production of charged pions, kaons and protons at large transverse momenta in pp and $\mathrm{Pb}-\mathrm{Pb}$ collisions at $\sqrt{s_{\mathrm{NN}}}=2.76 \mathrm{TeV}$, Phys. Lett. B 736 (2014) 196 [arXiv: 1401.1250] [INSPIRE].

[68] ALICE collaboration, Production of $\pi^{0}$ and $\eta$ mesons up to high transverse momentum in pp collisions at 2.76 TeV, Eur. Phys. J. C 77 (2017) 339 [arXiv:1702.00917] [INSPIRE].

[69] WA80 collaboration, R. Albrecht et al., Production of eta mesons in 200- $\mathrm{A} / \mathrm{GeV} S+S$ and $S+$ Au reactions, Phys. Lett. B 361 (1995) 14 [hep-ex/9507009] [INSPIRE].

[70] P.K. Khandai, P. Shukla and V. Singh, Meson spectra and $m_{T}$ scaling in $p+p, d+A u$ and Au + Au collisions at $\sqrt{s_{\mathrm{NN}}}=200 \mathrm{GeV}$, Phys. Rev. C 84 (2011) 054904 [arXiv:1110.3929] [INSPIRE].

[71] ALICE collaboration, Neutral pion and $\eta$ meson production at mid-rapidity in $\mathrm{Pb}-\mathrm{Pb}$ collisions at $\sqrt{s_{\mathrm{NN}}}=2.76 \mathrm{TeV}$, arXiv: 1803.05490 [INSPIRE]. 
[72] F. Bossu, Z.C. del Valle, A. de Falco, M. Gagliardi, S. Grigoryan and G. Martinez Garcia, Phenomenological interpolation of the inclusive $J / \psi$ cross section to proton-proton collisions at 2.76 TeV and 5.5 TeV, arXiv:1103.2394 [INSPIRE].

[73] ALICE collaboration, Inclusive, prompt and non-prompt $J / \psi$ production at mid-rapidity in $\mathrm{Pb}-\mathrm{Pb}$ collisions at $\sqrt{s_{\mathrm{NN}}}=2.76 \mathrm{TeV}, \mathrm{JHEP} 07$ (2015) 051 [arXiv:1504.07151] [INSPIRE].

[74] ALICE collaboration, $J / \psi$ suppression at forward rapidity in $\mathrm{Pb}-\mathrm{Pb}$ collisions at $\sqrt{s_{\mathrm{NN}}}=5.02 \mathrm{TeV}$, Phys. Lett. B 766 (2017) 212 [arXiv:1606.08197] [INSPIRE].

[75] ALICE collaboration, Measurement of inelastic, single- and double-diffraction cross sections in proton-proton collisions at the LHC with ALICE, Eur. Phys. J. C 73 (2013) 2456 [arXiv: 1208.4968] [INSPIRE].

[76] ALICE collaboration, Centrality Dependence of Charged Particle Production at Large Transverse Momentum in Pb-Pb Collisions at $\sqrt{s_{\mathrm{NN}}}=2.76 \mathrm{TeV}$, Phys. Lett. B 720 (2013) 52 [arXiv: 1208.2711] [INSPIRE].

[77] M. Cacciari, M. Greco and P. Nason, The $p_{T}$ spectrum in heavy flavor hadroproduction, JHEP 05 (1998) 007 [hep-ph/9803400] [INSPIRE].

[78] P.M. Nadolsky et al., Implications of CTEQ global analysis for collider observables, Phys. Rev. D 78 (2008) 013004 [arXiv: 0802.0007] [INSPIRE].

[79] CDF collaboration, D. Acosta et al., Measurement of prompt charm meson production cross sections in p p collisions at $\sqrt{s}=1.96$ TeV, Phys. Rev. Lett. 91 (2003) 241804 [hep-ex/0307080] [INSPIRE].

[80] J. Uphoff, O. Fochler, Z. Xu and C. Greiner, Open Heavy Flavor in $\mathrm{Pb}-\mathrm{Pb}$ Collisions at $\sqrt{s}=2.76 \mathrm{TeV}$ within a Transport Model, Phys. Lett. B 717 (2012) 430 [arXiv:1205.4945] [INSPIRE].

[81] J. Uphoff, O. Fochler, Z. Xu and C. Greiner, Heavy vs. light flavor energy loss within a partonic transport model, J. Phys. Conf. Ser. 509 (2014) 012077 [arXiv:1310.3597] [INSPIRE].

[82] M. He, R.J. Fries and R. Rapp, Heavy Flavor at the Large Hadron Collider in a Strong Coupling Approach, Phys. Lett. B 735 (2014) 445 [arXiv:1401.3817] [INSPIRE].

[83] W.M. Alberico et al., Heavy flavors in AA collisions: production, transport and final spectra, Eur. Phys. J. C 73 (2013) 2481 [arXiv: 1305.7421] [InSPIRE].

[84] M. Nahrgang, J. Aichelin, P.B. Gossiaux and K. Werner, Influence of hadronic bound states above $T_{c}$ on heavy-quark observables in $\mathrm{Pb}-\mathrm{Pb}$ collisions at the CERN Large Hadron Collider, Phys. Rev. C 89 (2014) 014905 [arXiv:1305.6544] [InSPIRE].

[85] S. Cao, G.-Y. Qin and S.A. Bass, Heavy-quark dynamics and hadronization in ultrarelativistic heavy-ion collisions: Collisional versus radiative energy loss, Phys. Rev. C 88 (2013) 044907 [arXiv: 1308.0617] [INSPIRE]. 


\section{The ALICE collaboration}

S. Acharya ${ }^{139}$, F.T.-. Acosta ${ }^{20}$, D. Adamováa3 ${ }^{93}$ J. Adolfsson ${ }^{80}$, M.M. Aggarwal ${ }^{98}$, G. Aglieri

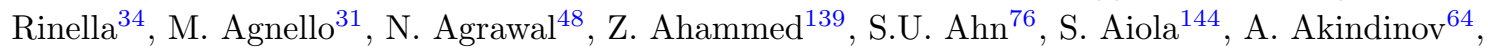
M. Al-Turany ${ }^{104}$, S.N. Alam ${ }^{139}$, D.S.D. Albuquerque ${ }^{121}$, D. Aleksandrov ${ }^{87}$, B. Alessandro ${ }^{58}$, R. Alfaro Molina ${ }^{72}$, Y. Ali ${ }^{15}$, A. Alici ${ }^{10,53,27}$, A. Alkin ${ }^{2}$, J. Alme ${ }^{22}$, T. Alt ${ }^{69}$, L. Altenkamper ${ }^{22}$, I. Altsybeev ${ }^{111}$, M.N. Anaam ${ }^{6}$, C. Andrei ${ }^{47}$, D. Andreou ${ }^{34}$, H.A. Andrews ${ }^{108}$, A. Andronic ${ }^{142,104}$, M. Angeletti ${ }^{34}$, V. Anguelov ${ }^{102}$, C. Anson ${ }^{16}$, T. Antičić ${ }^{105}$, F. Antinori ${ }^{56}$, P. Antonioli ${ }^{53}$, R. Anwar ${ }^{125}$, N. Apadula ${ }^{79}$, L. Aphecetche ${ }^{113}$, H. Appelshäuser ${ }^{69}$, S. Arcelli ${ }^{27}$, R. Arnaldi ${ }^{58}$, O.W. Arnold ${ }^{103,116}$, I.C. Arsene ${ }^{21}$, M. Arslandok ${ }^{102}$, A. Augustinus ${ }^{34}$, R. Averbeck ${ }^{104}$,

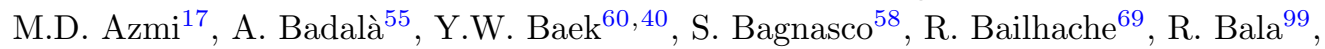
A. Baldisseri ${ }^{135}$, M. Ball ${ }^{42}$, R.C. Baral ${ }^{85}$, A.M. Barbano ${ }^{26}$, R. Barbera ${ }^{28}$, F. Barile ${ }^{52}$, L. Barioglio ${ }^{26}$, G.G. Barnaföldi ${ }^{143}$, L.S. Barnby ${ }^{92}$, V. Barret ${ }^{132}$, P. Bartalini ${ }^{6}$, K. Barth ${ }^{34}$, E. Bartsch ${ }^{69}$, N. Bastid ${ }^{132}$, S. Basu ${ }^{141}$, G. Batigne ${ }^{113}$, B. Batyunya ${ }^{75}$, P.C. Batzing ${ }^{21}$, J.L. Bazo Alba ${ }^{109}$, I.G. Bearden ${ }^{88}$, H. Beck ${ }^{102}$, C. Bedda ${ }^{63}$, N.K. Behera ${ }^{60}$, I. Belikov ${ }^{134}$, F. Bellini ${ }^{34}$, H. Bello Martinez ${ }^{44}$, R. Bellwied ${ }^{125}$, L.G.E. Beltran ${ }^{119}$, V. Belyaev ${ }^{91}$, G. Bencedi ${ }^{143}$, S. Beole ${ }^{26}$, A. Bercuci ${ }^{47}$, Y. Berdnikov ${ }^{96}$, D. Berenyi ${ }^{143}$, R.A. Bertens ${ }^{128}$, D. Berzano ${ }^{34,58}$, L. Betev ${ }^{34}$, P.P. Bhaduri ${ }^{139}$, A. Bhasin ${ }^{99}$, I.R. Bhat ${ }^{99}$, H. Bhatt ${ }^{48}$, B. Bhattacharjee ${ }^{41}$, J. Bhom ${ }^{117}$, A. Bianchi ${ }^{26}$, L. Bianchi ${ }^{125}$, N. Bianchi ${ }^{51}$, J. Bielčík ${ }^{37}$, J. Bielčíkováa3 ${ }^{93}$

A. Bilandzic ${ }^{116,103}$, G. Biro ${ }^{143}$, R. Biswas ${ }^{3}$, S. Biswas ${ }^{3}$, J.T. Blair ${ }^{118}$, D. Blau ${ }^{87}$, C. Blume ${ }^{69}$, G. Boca ${ }^{137}$, F. Bock ${ }^{34}$, A. Bogdanov ${ }^{91}$, L. Boldizsár ${ }^{143}$, M. Bombara $^{38}$, G. Bonomi $^{138}$, M. Bonora ${ }^{34}$, H. Borel ${ }^{135}$, A. Borissov ${ }^{142}$, M. Borri ${ }^{127}$, E. Botta ${ }^{26}$, C. Bourjau ${ }^{88}$, L. Bratrud ${ }^{69}$, P. Braun-Munzinger ${ }^{104}$, M. Bregant ${ }^{120}$, T.A. Broker ${ }^{69}$, M. Broz ${ }^{37}$, E.J. Brucken ${ }^{43}$, E. Bruna ${ }^{58}$, G.E. Bruno ${ }^{34,33}$, D. Budnikov ${ }^{106}$, H. Buesching ${ }^{69}$, S. Bufalino ${ }^{31}$, P. Buhler ${ }^{112}$, P. Buncic ${ }^{34}$, O. Busch ${ }^{131}$, i, Z. Buthelezi ${ }^{73}$, J.B. Butt ${ }^{15}$, J.T. Buxton ${ }^{95}$, J. Cabala ${ }^{115}$, D. Caffarri ${ }^{89}$,

H. Caines ${ }^{144}$, A. Caliva ${ }^{104}$, E. Calvo Villar ${ }^{109}$, R.S. Camacho ${ }^{44}$, P. Camerini ${ }^{25}$, A.A. Capon ${ }^{112}$, F. Carena ${ }^{34}$, W. Carena ${ }^{34}$, F. Carnesecchi ${ }^{27,10}$, J. Castillo Castellanos ${ }^{135}$, A.J. Castro ${ }^{128}$, E.A.R. Casula ${ }^{54}$, C. Ceballos Sanchez ${ }^{8}$, S. Chandra ${ }^{139}$, B. Chang ${ }^{126}$, W. Chang ${ }^{6}$, S. Chapeland ${ }^{34}$, M. Chartier ${ }^{127}$, S. Chattopadhyay ${ }^{139}$, S. Chattopadhyay ${ }^{107}$, A. Chauvin ${ }^{103,116}$, C. Cheshkov ${ }^{133}$, B. Cheynis ${ }^{133}$, V. Chibante Barroso ${ }^{34}$, D.D. Chinellato ${ }^{121}$, S. Cho ${ }^{60}$, P. Chochula ${ }^{34}$, T. Chowdhury ${ }^{132}$, P. Christakoglou ${ }^{89}$, C.H. Christensen ${ }^{88}$, P. Christiansen ${ }^{80}$, T. Chujo $^{131}$, S.U. Chung ${ }^{18}$, C. Cicalo ${ }^{54}$, L. Cifarelli ${ }^{10,27}$, F. Cindolo ${ }^{53}$, J. Cleymans $^{124}$, F. Colamaria ${ }^{52}$, D. Colella ${ }^{65,52}$, A. Collu ${ }^{79}$, M. Colocci ${ }^{27}$, M. Concas ${ }^{58, ~ i i}$, G. Conesa Balbastre ${ }^{78}$, Z. Conesa del Valle $^{61}$, J.G. Contreras ${ }^{37}$, T.M. Cormier ${ }^{94}$, Y. Corrales Morales ${ }^{58}$, P. Cortese $^{32}$, M.R. Cosentino ${ }^{122}$, F. Costa ${ }^{34}$, S. Costanza ${ }^{137}$, J. Crkovská6 ${ }^{61}$, P. Crochet $^{132}$, E. Cuautle ${ }^{70}$, L. Cunqueiro ${ }^{142,94}$, T. Dahms ${ }^{103,116}$, A. Dainese ${ }^{56}$, S. Dani ${ }^{66}$, M.C. Danisch ${ }^{102}$, A. Danu ${ }^{68}$,

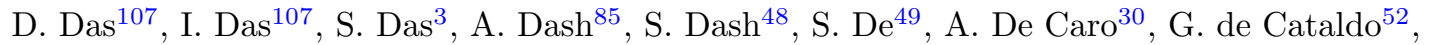
C. de Conti ${ }^{120}$, J. de Cuveland ${ }^{39}$, A. De Falco ${ }^{24}$, D. De Gruttola ${ }^{10,30}$, N. De Marco ${ }^{58}$, S. De Pasquale $^{30}$, R.D. De Souza ${ }^{121}$, H.F. Degenhardt ${ }^{120}$, A. Deisting ${ }^{104,102}$, A. Deloff ${ }^{84}$, S. Delsanto ${ }^{26}$, C. Deplano ${ }^{89}$, P. Dhankher ${ }^{48}$, D. Di Bari ${ }^{33}$, A. Di Mauro ${ }^{34}$, B. Di Ruzza ${ }^{56}$, R.A. Diaz ${ }^{8}$, T. Dietel ${ }^{124}$, P. Dillenseger ${ }^{69}$, Y. Ding ${ }^{6}$, R. Divià ${ }^{34}$, Ø. Djuvsland ${ }^{22}$, A. Dobrin ${ }^{34}$, D. Domenicis Gimenez $^{120}$, B. Dönigus ${ }^{69}$, O. Dordic ${ }^{21}$, L.V.R. Doremalen ${ }^{63}$, A.K. Dubey ${ }^{139}$, A. Dubla ${ }^{104}$, L. Ducroux ${ }^{133}$, S. Dudi ${ }^{98}$, A.K. Duggal ${ }^{98}$, M. Dukhishyam ${ }^{85}$, P. Dupieux ${ }^{132}$, R.J. Ehlers ${ }^{144}$, D. Elia ${ }^{52}$, E. Endress ${ }^{109}$, H. Engel ${ }^{74}$, E. Epple ${ }^{144}$, B. Erazmus ${ }^{113}$, F. Erhardt ${ }^{97}$, M.R. Ersdal ${ }^{22}$, B. Espagnon ${ }^{61}$, G. Eulisse ${ }^{34}$, J. Eum ${ }^{18}$, D. Evans ${ }^{108}$, S. Evdokimov ${ }^{90}$, L. Fabbietti ${ }^{103,116}$, M. Faggin ${ }^{29}$, J. Faivre ${ }^{78}$, A. Fantoni ${ }^{51}$, M. Fasel ${ }^{94}$, L. Feldkamp ${ }^{142}$, A. Feliciello ${ }^{58}$, G. Feofilov ${ }^{111}$, A. Fernández Téllez ${ }^{44}$, A. Ferretti ${ }^{26}$, A. Festanti ${ }^{34}$, V.J.G. Feuillard ${ }^{102}$, J. Figiel ${ }^{117}$, M.A.S. Figueredo ${ }^{120}$, S. Filchagin ${ }^{106}$, D. Finogeev ${ }^{62}$, F.M. Fionda ${ }^{22}$, G. Fiorenza ${ }^{52}$, F. Flor ${ }^{125}$, 
M. Floris ${ }^{34}$, S. Foertsch ${ }^{73}$, P. Foka ${ }^{104}$, S. Fokin ${ }^{87}$, E. Fragiacomo ${ }^{59}$, A. Francescon ${ }^{34}$, A. Francisco ${ }^{113}$, U. Frankenfeld ${ }^{104}$, G.G. Fronze ${ }^{26}$, U. Fuchs ${ }^{34}$, C. Furget ${ }^{78}$, A. Furs ${ }^{62}$, M. Fusco Girard $^{30}$, J.J. Gaardhøje ${ }^{88}$, M. Gagliardi ${ }^{26}$, A.M. Gago ${ }^{109}$, K. Gajdosova ${ }^{88}$, M. Gallio ${ }^{26}$, C.D. Galvan ${ }^{119}$, P. Ganoti ${ }^{83}$, C. Garabatos ${ }^{104}$, E. Garcia-Solis ${ }^{11}$, K. Garg ${ }^{28}$, C. Gargiulo ${ }^{34}$, P. Gasik ${ }^{116,103}$, E.F. Gauger ${ }^{118}$, M.B. Gay Ducati ${ }^{71}$, M. Germain ${ }^{113}$, J. Ghosh ${ }^{107}$, P. Ghosh ${ }^{139}$, S.K. Ghosh ${ }^{3}$, P. Gianotti ${ }^{51}$, P. Giubellino ${ }^{104,58}$, P. Giubilato ${ }^{29}$, P. Glässel ${ }^{102}$, D.M. Goméz $\mathrm{Coral}^{72}$, A. Gomez Ramirez ${ }^{74}$, V. Gonzalez ${ }^{104}$, P. González-Zamora ${ }^{44}$, S. Gorbunov ${ }^{39}$, L. Görlich ${ }^{117}$, S. Gotovac ${ }^{35}$, V. Grabskii ${ }^{72}$, L.K. Graczykowski ${ }^{140}$, K.L. Graham ${ }^{108}$, L. Greiner ${ }^{79}$, A. Grelli63 ${ }^{63}$ C. Grigoras $^{34}$, V. Grigoriev ${ }^{91}$, A. Grigoryan ${ }^{1}$, S. Grigoryan ${ }^{75}$, J.M. Gronefeld ${ }^{104}$, F. Grosa ${ }^{31}$, J.F. Grosse-Oetringhaus ${ }^{34}$, R. Grosso ${ }^{104}$, R. Guernane ${ }^{78}$, B. Guerzoni ${ }^{27}$, M. Guittiere ${ }^{113}$, K. Gulbrandsen ${ }^{88}$, T. Gunji ${ }^{130}$, A. Gupta ${ }^{99}$, R. Gupta ${ }^{99}$, I.B. Guzman ${ }^{44}$, R. Haake ${ }^{34}$, M.K. Habib ${ }^{104}$, C. Hadjidakis ${ }^{61}$, H. Hamagaki ${ }^{81}$, G. Hamar ${ }^{143}$, M. Hamid ${ }^{6}$, J.C. Hamon ${ }^{134}$, R. Hannigan ${ }^{118}$, M.R. Haque ${ }^{63}$, A. Harlenderova ${ }^{104}$, J.W. Harris ${ }^{144}$, A. Harton ${ }^{11}$, H. Hassan ${ }^{78}$, D. Hatzifotiadou ${ }^{53,10}$, S. Hayashi ${ }^{130}$, S.T. Heckel ${ }^{69}$, E. Hellbär ${ }^{69}$, H. Helstrup ${ }^{36}$, A. Herghelegiu ${ }^{47}$, E.G. Hernandez ${ }^{44}$, G. Herrera Corral ${ }^{9}$, F. Herrmann ${ }^{142}$, K.F. Hetland ${ }^{36}$, T.E. Hilden ${ }^{43}$, H. Hillemanns ${ }^{34}$, C. Hills ${ }^{127}$, B. Hippolyte ${ }^{134}$, B. Hohlweger ${ }^{103}$, D. Horak ${ }^{37}$,

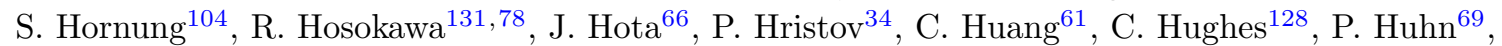
T.J. Humanic ${ }^{95}$, H. Hushnud ${ }^{107}$, N. Hussain ${ }^{41}$, T. Hussain ${ }^{17}$, D. Hutter ${ }^{39}$, D.S. Hwang ${ }^{19}$, J.P. Iddon ${ }^{127}$, S.A. Iga Buitron ${ }^{70}$, R. Ilkaev ${ }^{106}$, M. Inaba ${ }^{131}$, M. Ippolitov ${ }^{87}$, M.S. Islam ${ }^{107}$, M. Ivanov ${ }^{104}$, V. Ivanov ${ }^{96}$, V. Izucheev ${ }^{90}$, B. Jacak ${ }^{79}$, N. Jacazio ${ }^{27}$, P.M. Jacobs ${ }^{79}$, M.B. Jadhav ${ }^{48}$, S. Jadlovska ${ }^{115}$, J. Jadlovsky ${ }^{115}$, S. Jaelani ${ }^{63}$, C. Jahnke ${ }^{120,116}$, M.J. Jakubowska ${ }^{140}$, M.A. Janik ${ }^{140}$, C. Jena ${ }^{85}$, M. Jercic ${ }^{97}$, O. Jevons ${ }^{108}$, R.T. Jimenez Bustamante $^{104}$, M. Jin ${ }^{125}$, P.G. Jones ${ }^{108}$, A. Jusko ${ }^{108}$, P. Kalinak ${ }^{65}$, A. Kalweit ${ }^{34}$, J.H. Kang ${ }^{145}$, V. Kaplin ${ }^{91}$, S. $\operatorname{Kar}^{6}$, A. Karasu Uysal ${ }^{77}$, O. Karavichev ${ }^{62}$, T. Karavicheva ${ }^{62}$, P. Karczmarczyk ${ }^{34}$, E. Karpechev ${ }^{62}$, U. Kebschull ${ }^{74}$, R. Keidel ${ }^{46}$, D.L.D. Keijdener ${ }^{63}$, M. Keil ${ }^{34}$, B. Ketzer ${ }^{42}$, Z. Khabanova ${ }^{89}$, A.M. Khan ${ }^{6}$, S. Khan ${ }^{17}$, S.A. Khan ${ }^{139}$, A. Khanzadeev ${ }^{96}$, Y. Kharlov ${ }^{90}$, A. Khatun ${ }^{17}$, A. Khuntia ${ }^{49}$, M.M. Kielbowicz ${ }^{117}$, B. Kileng ${ }^{36}$, B. Kim ${ }^{131}$, D. Kim ${ }^{145}$, D.J. Kim ${ }^{126}$, E.J. $\operatorname{Kim}^{13}$, H. Kim ${ }^{145}$, J.S. $\operatorname{Kim}^{40}$, J. $\operatorname{Kim}^{102}$, M. $\operatorname{Kim}^{102,60}$, S. Kim ${ }^{19}$, T. $\operatorname{Kim}^{145}$, T. $\operatorname{Kim}^{145}$, S. Kirsch ${ }^{39}$, I. Kisel ${ }^{39}$, S. Kiselev ${ }^{64}$, A. Kisiel ${ }^{140}$, J.L. Klay ${ }^{5}$, C. Klein ${ }^{69}$, J. Klein ${ }^{34,58}$, C. Klein-Bösing ${ }^{142}$, S. Klewin ${ }^{102}$, A. Kluge ${ }^{34}$, M.L. Knichel ${ }^{34}$, A.G. Knospe ${ }^{125}$, C. Kobdaj ${ }^{114}$, M. Kofarago ${ }^{143}$, M.K. Köhler ${ }^{102}$, T. Kollegger ${ }^{104}$, N. Kondratyeva ${ }^{91}$, E. Kondratyuk ${ }^{90}$, A. Konevskikh ${ }^{62}$, P.J. Konopka ${ }^{34}$, M. Konyushikhin ${ }^{141}$, O. Kovalenko ${ }^{84}$, V. Kovalenko ${ }^{111}$, M. Kowalskii ${ }^{117}$, I. Králik ${ }^{65}$, A. Kravčákováa ${ }^{38}$, L. Kreis ${ }^{104}$, M. Krivda ${ }^{65,108}$, F. Krizek ${ }^{93}$, M. Krüger ${ }^{69}$, E. Kryshen ${ }^{96}$, M. Krzewicki ${ }^{39}$, A.M. Kubera ${ }^{95}$, V. Kučera ${ }^{60,93}$, C. Kuhn ${ }^{134}$, P.G. Kuijer ${ }^{89}$, J. Kumar ${ }^{48}$, L. Kumar ${ }^{98}$, S. Kumar ${ }^{48}$, S. Kundu ${ }^{85}$, P. Kurashvili ${ }^{84}$, A. Kurepin ${ }^{62}$, A.B. Kurepin ${ }^{62}$, A. Kuryakin ${ }^{106}$, S. Kushpil ${ }^{93}$, J. Kvapil ${ }^{108}$, M.J. Kweon ${ }^{60}$, Y. Kwon ${ }^{145}$, S.L. La

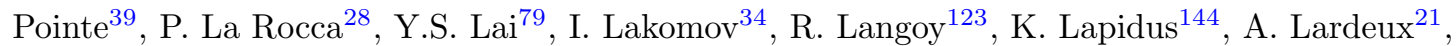
P. Larionov ${ }^{51}$, E. Laudi ${ }^{34}$, R. Lavicka ${ }^{37}$, R. Lea ${ }^{25}$, L. Leardini ${ }^{102}$, S. Lee ${ }^{145}$, F. Lehas ${ }^{89}$, S. Lehner ${ }^{112}$, J. Lehrbach ${ }^{39}$, R.C. Lemmon ${ }^{92}$, I. León Monzón ${ }^{119}$, P. Lévai ${ }^{143}$, X. Li $^{12}$, X.L. Li ${ }^{6}$, J. Lien ${ }^{123}$, R. Lietava ${ }^{108}$, B. $\operatorname{Lim}^{18}$, S. Lindal ${ }^{21}$, V. Lindenstruth ${ }^{39}$, S.W. Lindsay ${ }^{127}$, C. Lippmann ${ }^{104}$, M.A. Lisa ${ }^{95}$, V. Litichevskyi ${ }^{43}$, A. Liu ${ }^{79}$, H.M. Ljunggren ${ }^{80}$, W.J. Llope ${ }^{141}$, D.F. Lodato ${ }^{63}$, V. Loginov $^{91}$, C. Loizides ${ }^{94,79}$, P. Loncar ${ }^{35}$, X. Lopez ${ }^{132}$, E. López Torres ${ }^{8}$, A. Lowe ${ }^{143}$, P. Luettig ${ }^{69}$, J.R. Luhder ${ }^{142}$, M. Lunardon ${ }^{29}$, G. Luparello ${ }^{59}$, M. Lupi ${ }^{34}$, A. Maevskaya ${ }^{62}$, M. Mager ${ }^{34}$, S.M. Mahmood ${ }^{21}$, A. Maire ${ }^{134}$, R.D. Majka ${ }^{144}$, M. Malaev ${ }^{96}$, Q.W. Malik ${ }^{21}$, L. Malinina ${ }^{75}$, iii, D. Mal'Kevich ${ }^{64}$, P. Malzacher ${ }^{104}$, A. Mamonov ${ }^{106}$, V. Manko ${ }^{87}$, F. Manso ${ }^{132}$, V. Manzari ${ }^{52}$, Y. Mao ${ }^{6}$, M. Marchisone ${ }^{129,73,133}$, J. Mareš ${ }^{67}$, G.V. Margagliotti ${ }^{25}$, A. Margotti ${ }^{53}$, J. Margutti ${ }^{63}$, A. Marín ${ }^{104}$, C. Markert ${ }^{118}$, M. Marquard ${ }^{69}$, N.A. Martin ${ }^{104}$, P. Martinengo ${ }^{34}$, J.L. Martinez ${ }^{125}$, M.I. Martínez ${ }^{44}$, G. Martínez García ${ }^{113}$, M. Martinez 
Pedreira $^{34}$, S. Masciocchi ${ }^{104}$, M. Masera ${ }^{26}$, A. Masoni ${ }^{54}$, L. Massacrier ${ }^{61}$, E. Masson $^{113}$, A. Mastroserio ${ }^{52,136}$, A.M. Mathis ${ }^{116,103}$, P.F.T. Matuoka ${ }^{120}$, A. Matyja ${ }^{117,128}$, C. Mayer $^{117}$, M. Mazzilli ${ }^{33}$, M.A. Mazzoni ${ }^{57}$, F. Meddi ${ }^{23}$, Y. Melikyan ${ }^{91}$, A. Menchaca-Rocha ${ }^{72}$, E. Meninno ${ }^{30}$, J. Mercado Pérez ${ }^{102}$, M. Meres ${ }^{14}$, C.S. Meza ${ }^{109}$, S. Mhlanga ${ }^{124}$, Y. Miake ${ }^{131}$, L. Micheletti ${ }^{26}$, M.M. Mieskolainen ${ }^{43}$, D.L. Mihaylov ${ }^{103}$, K. Mikhaylov ${ }^{64} 75$, A. Mischke ${ }^{63}$, A.N. Mishra ${ }^{70}$, D. Miśkowiec ${ }^{104}$, J. Mitra ${ }^{139}$, C.M. Mitu ${ }^{68}$, N. Mohammadi ${ }^{34}$, A.P. Mohanty ${ }^{63}$, B. Mohanty ${ }^{85}$, M. Mohisin Khan ${ }^{17}$, iv , D.A. Moreira De Godoy ${ }^{142}$, L.A.P. Moreno ${ }^{44}$, S. Moretto ${ }^{29}$,

A. Morreale ${ }^{113}$, A. Morsch ${ }^{34}$, T. Mrnjavac $^{34}$, V. Muccifora ${ }^{51}$, E. Mudnic ${ }^{35}$, D. Mühlheim ${ }^{142}$, S. Muhuri ${ }^{139}$, M. Mukherjee ${ }^{3}$, J.D. Mulligan ${ }^{144}$, M.G. Munhoz ${ }^{120}$, K. Münning ${ }^{42}$, M.I.A. Munoz ${ }^{79}$, R.H. Munzer ${ }^{69}$, H. Murakami ${ }^{130}$, S. Murray ${ }^{73}$, L. Musa ${ }^{34}$, J. Musinsky ${ }^{65}$, C.J. Myers ${ }^{125}$,

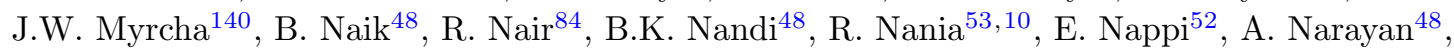
M.U. Naru ${ }^{15}$, A.F. Nassirpour ${ }^{80}$, H. Natal da Luz ${ }^{120}$, C. Nattrass ${ }^{128}$, S.R. Navarro ${ }^{44}$, K. Nayak ${ }^{85}$, R. Nayak $^{48}$, T.K. Nayak ${ }^{139}$, S. Nazarenko ${ }^{106}$, R.A. Negrao De Oliveira ${ }^{69,34}$, L. Nellen ${ }^{70}$, S.V. Nesbo ${ }^{36}$, G. Neskovic ${ }^{39}$, F. $\mathrm{Ng}^{125}$, M. Nicassio ${ }^{104}$, J. Niedziela ${ }^{140,34}$, B.S. Nielsen ${ }^{88}$, S. Nikolaev ${ }^{87}$, S. Nikulin ${ }^{87}$, V. Nikulin ${ }^{96}$, F. Noferini ${ }^{10,53}$, P. Nomokonov ${ }^{75}$, G. Nooren ${ }^{63}$, J.C.C. Noris ${ }^{44}$, J. Norman ${ }^{78}$, A. Nyanin ${ }^{87}$, J. Nystrand ${ }^{22}$, H. Oh ${ }^{145}$, A. Ohlson ${ }^{102}$, J. Oleniacz ${ }^{140}$, A.C. Oliveira Da Silva ${ }^{120}$, M.H. Oliver ${ }^{144}$, J. Onderwaater ${ }^{104}$, C. Oppedisano ${ }^{58}$, R. Orava ${ }^{43}$, M. Oravec ${ }^{115}$, A. Ortiz Velasquez ${ }^{70}$, A. Oskarsson ${ }^{80}$, J. Otwinowski ${ }^{117}$, K. Oyama ${ }^{81}$, Y. Pachmayer ${ }^{102}$, V. Pacik ${ }^{88}$, D. Pagano ${ }^{138}$, G. Paić ${ }^{70}$, P. Palni ${ }^{6}$, J. Pan ${ }^{141}$, A.K. Pandey ${ }^{48}$, S. Panebianco ${ }^{135}$, V. Papikyan ${ }^{1}$, P. Pareek ${ }^{49}$, J. Park ${ }^{60}$, J.E. Parkkila ${ }^{126}$, S. Parmar ${ }^{98}$, A. Passfeld ${ }^{142}$, S.P. Pathak ${ }^{125}$, R.N. Patra ${ }^{139}$, B. Paul ${ }^{58}$, H. Pei ${ }^{6}$, T. Peitzmann ${ }^{63}$, X. Peng ${ }^{6}$, L.G. Pereira ${ }^{71}$, H. Pereira Da Costa ${ }^{135}$, D. Peresunko ${ }^{87}$, E. Perez Lezama ${ }^{69}$, V. Peskov ${ }^{69}$, Y. Pestov ${ }^{4}$, V. Petráček ${ }^{37}$, M. Petrovici ${ }^{47}$, C. Petta ${ }^{28}$, R.P. Pezzi ${ }^{71}$, S. Piano ${ }^{59}$, M. Pikna ${ }^{14}$, P. Pillot ${ }^{113}$, L.O.D.L. Pimentel ${ }^{88}$, O. Pinazza ${ }^{53,34}$, L. Pinsky ${ }^{125}$, S. Pisano ${ }^{51}$, D.B. Piyarathna ${ }^{125}$, M. Płoskoń ${ }^{79}$, M. Planinic ${ }^{97}$, F. Pliquett ${ }^{69}$, J. Pluta ${ }^{140}$, S. Pochybova $^{143}$,

P.L.M. Podesta-Lerma ${ }^{119}$, M.G. Poghosyan ${ }^{94}$, B. Polichtchouk ${ }^{90}$, N. Poljak ${ }^{97}$, W. Poonsawat ${ }^{114}$, A. Pop ${ }^{47}$, H. Poppenborg ${ }^{142}$, S. Porteboeuf-Houssais ${ }^{132}$, V. Pozdniakov ${ }^{75}$, S.K. Prasad ${ }^{3}$, R. Preghenella ${ }^{53}$, F. Prino ${ }^{58}$, C.A. Pruneau ${ }^{141}$, I. Pshenichnov ${ }^{62}$, M. Puccio $^{26}$, V. Punin ${ }^{106}$, J. Putschke ${ }^{141}$, S. Raha ${ }^{3}$, S. Rajput ${ }^{99}$, J. Rak ${ }^{126}$, A. Rakotozafindrabe ${ }^{135}$, L. Ramello ${ }^{32}$, F. Rami ${ }^{134}$, R. Raniwala ${ }^{100}$, S. Raniwala ${ }^{100}$, S.S. Räsänen ${ }^{43}$, B.T. Rascanu ${ }^{69}$, V. Ratza ${ }^{42}$ I. Ravasenga ${ }^{31}$, K.F. Read ${ }^{128,94}$, K. Redlich ${ }^{84, v}$, A. Rehman ${ }^{22}$, P. Reichelt ${ }^{69}$, F. Reidt ${ }^{34}$, X. Ren $^{6}$, R. Renfordt ${ }^{69}$, A. Reshetin ${ }^{62}$, J.-P. Revol ${ }^{10}$, K. Reygers ${ }^{102}$, V. Riabov ${ }^{96}$, T. Richert ${ }^{63}$, M. Richter ${ }^{21}$, P. Riedler ${ }^{34}$, W. Riegler ${ }^{34}$, F. Riggi ${ }^{28}$, C. Ristea ${ }^{68}$, S.P. Rode ${ }^{49}$, M. Rodríguez Cahuantzi $^{44}$, K. Røed ${ }^{21}$, R. Rogalev ${ }^{90}$, E. Rogochaya ${ }^{75}$, D. Rohr ${ }^{34}$, D. Röhrich ${ }^{22}$, P.S. Rokita ${ }^{140}$, F. Ronchetti ${ }^{51}$, E.D. Rosas ${ }^{70}$, K. Roslon ${ }^{140}$, P. Rosnet ${ }^{132}$, A. Rossi ${ }^{29}$, A. Rotondi ${ }^{137}$, F. Roukoutakis ${ }^{83}$, C. Roy ${ }^{134}$, P. Roy ${ }^{107}$, O.V. Rueda ${ }^{70}$, R. Rui ${ }^{25}$, B. Rumyantsev ${ }^{75}$, A. Rustamov ${ }^{86}$, E. Ryabinkin ${ }^{87}$, Y. Ryabov ${ }^{96}$, A. Rybicki ${ }^{117}$, S. Saarinen ${ }^{43}$, S. Sadhu ${ }^{139}$, S. Sadovsky ${ }^{90}$, K. Šafař́k ${ }^{34}$, S.K. Saha ${ }^{139}$, B. Sahoo ${ }^{48}$, P. Sahoo ${ }^{49}$, R. Sahoo ${ }^{49}$, S. Sahoo ${ }^{66}$, P.K. Sahu ${ }^{66}$, J. Saini ${ }^{139}$, S. Sakai ${ }^{131}$, M.A. Saleh ${ }^{141}$, S. Sambyal ${ }^{99}$, V. Samsonov ${ }^{96,91}$, A. Sandoval ${ }^{72}$, A. Sarkar ${ }^{73}$, D. Sarkar ${ }^{139}$, N. Sarkar ${ }^{139}$, P. Sarma ${ }^{41}$, M.H.P. Sas ${ }^{63}$, E. Scapparone ${ }^{53}$, F. Scarlassara ${ }^{29}$, B. Schaefer ${ }^{94}$, H.S. Scheid ${ }^{69}$, C. Schiaua ${ }^{47}$, R. Schicker ${ }^{102}$, C. Schmidt ${ }^{104}$, H.R. Schmidt ${ }^{101}$, M.O. Schmidt ${ }^{102}$, M. Schmidt ${ }^{101}$, N.V. Schmidt ${ }^{94,69}$, J. Schukraft ${ }^{34}$, Y. Schutz ${ }^{34,134}$, K. Schwarz ${ }^{104}$, K. Schweda ${ }^{104}$, G. Scioli ${ }^{27}$, E. Scomparin ${ }^{58}$, M. Šefčík ${ }^{38}$, J.E. Seger ${ }^{16}$, Y. Sekiguchi ${ }^{130}$, D. Sekihata ${ }^{45}$, I. Selyuzhenkov ${ }^{104,91}$, S. Senyukov ${ }^{134}$, E. Serradilla ${ }^{72}$, P. Sett ${ }^{48}$, A. Sevcenco ${ }^{68}$, A. Shabanov ${ }^{62}$, A. Shabetai ${ }^{113}$, R. Shahoyan ${ }^{34}$, W. Shaikh ${ }^{107}$, A. Shangaraev ${ }^{90}$, A. Sharma ${ }^{98}$, A. Sharma ${ }^{99}$, M. Sharma ${ }^{99}$, N. Sharma ${ }^{98}$, A.I. Sheikh ${ }^{139}$, K. Shigaki ${ }^{45}$, M. Shimomura ${ }^{82}$, S. Shirinkin ${ }^{64}$, Q. Shou ${ }^{6,110}$, K. Shtejer ${ }^{26}$, Y. Sibiriak ${ }^{87}$, S. Siddhanta ${ }^{54}$, K.M. Sielewicz ${ }^{34}$, T. Siemiarczuk ${ }^{84}$, D. Silvermyr ${ }^{80}$, G. Simatovic ${ }^{89}$, 
G. Simonetti ${ }^{34,103}$, R. Singaraju ${ }^{139}$, R. $\operatorname{Singh}^{85}$, R. Singh ${ }^{99}$, V. Singhal ${ }^{139}$, T. Sinha ${ }^{107}$, B. Sitar ${ }^{14}$, M. Sitta ${ }^{32}$, T.B. Skaali ${ }^{21}$, M. Slupecki ${ }^{126}$, N. Smirnov ${ }^{144}$, R.J.M. Snellings ${ }^{63}$, T.W. Snellman ${ }^{126}$, J. Song ${ }^{18}$, F. Soramel ${ }^{29}$, S. Sorensen ${ }^{128}$, F. Sozzi ${ }^{104}$, I. Sputowska ${ }^{117}$, J. Stachel ${ }^{102}$, I. Stan ${ }^{68}$, P. Stankus ${ }^{94}$, E. Stenlund ${ }^{80}$, D. Stocco ${ }^{113}$, M.M. Storetvedt ${ }^{36}$, P. Strmen ${ }^{14}$, A.A.P. Suaide ${ }^{120}$, T. Sugitate ${ }^{45}$, C. Suire ${ }^{61}$, M. Suleymanov ${ }^{15}$, M. Suljic ${ }^{34,25}$, R. Sultanov ${ }^{64}$, M. Sumbera ${ }^{93}$, S. Sumowidagdo ${ }^{50}$, K. Suzuki ${ }^{112}$, S. Swain ${ }^{66}$, A. Szabo ${ }^{14}$, I. Szarka ${ }^{14}$, U. Tabassam ${ }^{15}$, J. Takahashi ${ }^{121}$, G.J. Tambave ${ }^{22}$, N. Tanaka ${ }^{131}$, M. Tarhini ${ }^{113}$, M. Tariq ${ }^{17}$, M.G. Tarzila ${ }^{47}$, A. Tauro ${ }^{34}$, G. Tejeda Muñoz ${ }^{44}$, A. Telesca ${ }^{34}$, C. Terrevoli ${ }^{29}$, B. Teyssier ${ }^{133}$, D. Thakur ${ }^{49}$, S. Thakur ${ }^{139}$, D. Thomas ${ }^{118}$, F. Thoresen ${ }^{88}$, R. Tieulent ${ }^{133}$, A. Tikhonov ${ }^{62}$, A.R. Timmins ${ }^{125}$, A. Toia ${ }^{69}$, N. Topilskaya ${ }^{62}$, M. Toppi ${ }^{51}$, S.R. Torres ${ }^{119}$, S. Tripathy ${ }^{49}$, S. Trogolo ${ }^{26}$,

G. Trombetta ${ }^{33}$, L. Tropp ${ }^{38}$, V. Trubnikov $^{2}$, W.H. Trzaska ${ }^{126}$, T.P. Trzcinski ${ }^{140}$, B.A. Trzeciak ${ }^{63}$, T. Tsuji ${ }^{130}$, A. Tumkin ${ }^{106}$, R. Turrisi ${ }^{56}$, T.S. Tveter ${ }^{21}$, K. Ullaland ${ }^{22}$, E.N. Umaka ${ }^{125}$, A. Uras ${ }^{133}$, G.L. Usai ${ }^{24}$, A. Utrobicic ${ }^{97}$, M. Vala ${ }^{115}$, J.W. Van Hoorne ${ }^{34}$, M. van Leeuwen ${ }^{63}$, P. Vande

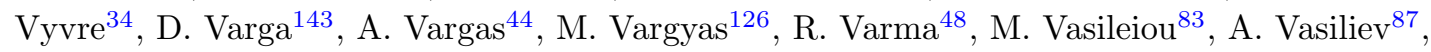
A. Vauthier ${ }^{78}$, O. Vázquez Doce ${ }^{103,116}$, V. Vechernin ${ }^{111}$, A.M. Veen ${ }^{63}$, E. Vercellin ${ }^{26}$, S. Vergara Limón $^{44}$, L. Vermunt ${ }^{63}$, R. Vernet ${ }^{7}$, R. Vértesi ${ }^{143}$, L. Vickovic ${ }^{35}$, J. Viinikainen ${ }^{126}$, Z. Vilakazi ${ }^{129}$, O. Villalobos Baillie ${ }^{108}$, A. Villatoro Tello ${ }^{44}$, A. Vinogradov ${ }^{87}$, T. Virgili ${ }^{30}$, V. Vislavicius ${ }^{88,80}$, A. Vodopyanov ${ }^{75}$, M.A. Völkl ${ }^{101}$, K. Voloshin ${ }^{64}$, S.A. Voloshin ${ }^{141}$, G. Volpe ${ }^{33}$, B. von Haller ${ }^{34}$, I. Vorobyev ${ }^{116,103}$, D. Voscek ${ }^{115}$, D. Vranic ${ }^{104,34}$, J. Vrláková ${ }^{38}$, B. Wagner ${ }^{22}$, H. Wang ${ }^{63}$, M. Wang 6 , Y. Watanabe ${ }^{131}$, M. Weber ${ }^{112}$, S.G. Weber ${ }^{104}$, A. Wegrzynek ${ }^{34}$, D.F. Weiser ${ }^{102}$, S.C. Wenzel ${ }^{34}$, J.P. Wessels ${ }^{142}$, U. Westerhoff ${ }^{142}$, A.M. Whitehead ${ }^{124}$, J. Wiechula ${ }^{69}$, J. Wikne ${ }^{21}$, G. Wilk ${ }^{84}$, J. Wilkinson ${ }^{53}$, G.A. Willems ${ }^{142,34}$, M.C.S. Williams ${ }^{53}$, E. Willsher ${ }^{108}$,

B. Windelband ${ }^{102}$, W.E. Witt ${ }^{128}$, R. Xu ${ }^{6}$, S. Yalcin ${ }^{77}$, K. Yamakawa ${ }^{45}$, S. Yano ${ }^{45}$, Z. Yin ${ }^{6}$,

H. Yokoyama ${ }^{78,131}$, I.-K. Yoo ${ }^{18}$, J.H. Yoon ${ }^{60}$, V. Yurchenko ${ }^{2}$, V. Zaccolo ${ }^{58}$, A. Zaman ${ }^{15}$,

C. Zampolli ${ }^{34}$, H.J.C. Zanoli ${ }^{120}$, N. Zardoshti ${ }^{108}$, A. Zarochentsev ${ }^{111}$, P. Závada ${ }^{67}$,

N. Zaviyalov ${ }^{106}$, H. Zbroszczyk ${ }^{140}$, M. Zhalov ${ }^{96}$, X. Zhang ${ }^{6}$, Y. Zhang ${ }^{6}$, Z. Zhang ${ }^{6,132}$, C. Zhao ${ }^{21}$, V. Zherebchevskii ${ }^{111}$, N. Zhigareva ${ }^{64}$, D. Zhou ${ }^{6}$, Y. Zhou ${ }^{88}$, Z. Zhou ${ }^{22}$, H. Zhu ${ }^{6}$, J. Zhu ${ }^{6}$, Y. Zhu ${ }^{6}$, A. Zichichi ${ }^{27,10}$, M.B. Zimmermann ${ }^{34}$, G. Zinovjev², J. Zmeskal ${ }^{112}$, S. Zou ${ }^{6}$,

i Deceased

ii Dipartimento DET del Politecnico di Torino, Turin, Italy

iii M.V. Lomonosov Moscow State University, D. V. Skobeltsyn Institute of Nuclear, Physics, Moscow, Russia

iv Department of Applied Physics, Aligarh Muslim University, Aligarh, India

$v$ Institute of Theoretical Physics, University of Wroclaw, Poland

1 A.I. Alikhanyan National Science Laboratory (Yerevan Physics Institute) Foundation, Yerevan, Armenia

2 Bogolyubov Institute for Theoretical Physics, National Academy of Sciences of Ukraine, Kiev, Ukraine

3 Bose Institute, Department of Physics and Centre for Astroparticle Physics and Space Science (CAPSS), Kolkata, India

4 Budker Institute for Nuclear Physics, Novosibirsk, Russia

5 California Polytechnic State University, San Luis Obispo, California, United States

6 Central China Normal University, Wuhan, China

7 Centre de Calcul de l'IN2P3, Villeurbanne, Lyon, France

8 Centro de Aplicaciones Tecnológicas y Desarrollo Nuclear (CEADEN), Havana, Cuba

9 Centro de Investigación y de Estudios Avanzados (CINVESTAV), Mexico City and Mérida, Mexico

10 Centro Fermi - Museo Storico della Fisica e Centro Studi e Ricerche "Enrico Fermi', Rome, Italy 
${ }^{3}$ Chonbuk National University, Jeonju, Republic of Korea

14 Comenius University Bratislava, Faculty of Mathematics, Physics and Informatics, Bratislava, Slovakia

15 COMSATS Institute of Information Technology (CIIT), Islamabad, Pakistan

16 Creighton University, Omaha, Nebraska, United States

17 Department of Physics, Aligarh Muslim University, Aligarh, India

18 Department of Physics, Pusan National University, Pusan, Republic of Korea

19 Department of Physics, Sejong University, Seoul, Republic of Korea

20 Department of Physics, University of California, Berkeley, California, United States

21 Department of Physics, University of Oslo, Oslo, Norway

${ }^{22}$ Department of Physics and Technology, University of Bergen, Bergen, Norway

23 Dipartimento di Fisica dell'Università 'La Sapienza' and Sezione INFN, Rome, Italy

24 Dipartimento di Fisica dell'Università and Sezione INFN, Cagliari, Italy

${ }^{25}$ Dipartimento di Fisica dell'Università and Sezione INFN, Trieste, Italy

26 Dipartimento di Fisica dell'Università and Sezione INFN, Turin, Italy

27 Dipartimento di Fisica e Astronomia dell'Università and Sezione INFN, Bologna, Italy

28 Dipartimento di Fisica e Astronomia dell'Università and Sezione INFN, Catania, Italy

${ }^{29}$ Dipartimento di Fisica e Astronomia dell'Università and Sezione INFN, Padova, Italy

${ }^{30}$ Dipartimento di Fisica 'E.R. Caianiello' dell'Università and Gruppo Collegato INFN, Salerno, Italy

31 Dipartimento DISAT del Politecnico and Sezione INFN, Turin, Italy

32 Dipartimento di Scienze e Innovazione Tecnologica dell'Università del Piemonte Orientale and INFN Sezione di Torino, Alessandria, Italy

33 Dipartimento Interateneo di Fisica 'M. Merlin' and Sezione INFN, Bari, Italy

34 European Organization for Nuclear Research (CERN), Geneva, Switzerland

${ }^{35}$ Faculty of Electrical Engineering, Mechanical Engineering and Naval Architecture, University of Split, Split, Croatia

36 Faculty of Engineering and Science, Western Norway University of Applied Sciences, Bergen, Norway

37 Faculty of Nuclear Sciences and Physical Engineering, Czech Technical University in Prague, Prague, Czech Republic

38 Faculty of Science, P.J. Šafárik University, Košice, Slovakia

39 Frankfurt Institute for Advanced Studies, Johann Wolfgang Goethe-Universität Frankfurt, Frankfurt, Germany

40 Gangneung-Wonju National University, Gangneung, Republic of Korea

41 Gauhati University, Department of Physics, Guwahati, India

${ }^{42}$ Helmholtz-Institut für Strahlen- und Kernphysik, Rheinische Friedrich-Wilhelms-Universität Bonn, Bonn, Germany

43 Helsinki Institute of Physics (HIP), Helsinki, Finland

${ }^{44}$ High Energy Physics Group, Universidad Autónoma de Puebla, Puebla, Mexico

45 Hiroshima University, Hiroshima, Japan

${ }^{46}$ Hochschule Worms, Zentrum für Technologietransfer und Telekommunikation (ZTT), Worms, Germany

47 Horia Hulubei National Institute of Physics and Nuclear Engineering, Bucharest, Romania

48 Indian Institute of Technology Bombay (IIT), Mumbai, India

49 Indian Institute of Technology Indore, Indore, India

50 Indonesian Institute of Sciences, Jakarta, Indonesia

51 INFN, Laboratori Nazionali di Frascati, Frascati, Italy

52 INFN, Sezione di Bari, Bari, Italy

53 INFN, Sezione di Bologna, Bologna, Italy

${ }^{54}$ INFN, Sezione di Cagliari, Cagliari, Italy 
${ }^{57}$ INFN, Sezione di Roma, Rome, Italy

58 INFN, Sezione di Torino, Turin, Italy

59 INFN, Sezione di Trieste, Trieste, Italy

60 Inha University, Incheon, Republic of Korea

${ }^{61}$ Institut de Physique Nucléaire d'Orsay (IPNO), Institut National de Physique Nucléaire et de Physique des Particules (IN2P3/CNRS), Université de Paris-Sud, Université Paris-Saclay, Orsay, France

62 Institute for Nuclear Research, Academy of Sciences, Moscow, Russia

63 Institute for Subatomic Physics, Utrecht University/Nikhef, Utrecht, Netherlands

${ }^{64}$ Institute for Theoretical and Experimental Physics, Moscow, Russia

65 Institute of Experimental Physics, Slovak Academy of Sciences, Košice, Slovakia

${ }^{66}$ Institute of Physics, Homi Bhabha National Institute, Bhubaneswar, India

${ }^{67}$ Institute of Physics of the Czech Academy of Sciences, Prague, Czech Republic

68 Institute of Space Science (ISS), Bucharest, Romania

69 Institut für Kernphysik, Johann Wolfgang Goethe-Universität Frankfurt, Frankfurt, Germany

70 Instituto de Ciencias Nucleares, Universidad Nacional Autónoma de México, Mexico City, Mexico

${ }^{71}$ Instituto de Física, Universidade Federal do Rio Grande do Sul (UFRGS), Porto Alegre, Brazil

${ }^{72}$ Instituto de Física, Universidad Nacional Autónoma de México, Mexico City, Mexico

73 iThemba LABS, National Research Foundation, Somerset West, South Africa

74 Johann-Wolfgang-Goethe Universität Frankfurt Institut für Informatik, Fachbereich Informatik und Mathematik, Frankfurt, Germany

75 Joint Institute for Nuclear Research (JINR), Dubna, Russia

76 Korea Institute of Science and Technology Information, Daejeon, Republic of Korea

77 KTO Karatay University, Konya, Turkey

78 Laboratoire de Physique Subatomique et de Cosmologie, Université Grenoble-Alpes, CNRS-IN2P3, Grenoble, France

79 Lawrence Berkeley National Laboratory, Berkeley, California, United States

80 Lund University Department of Physics, Division of Particle Physics, Lund, Sweden

81 Nagasaki Institute of Applied Science, Nagasaki, Japan

82 Nara Women's University (NWU), Nara, Japan

83 National and Kapodistrian University of Athens, School of Science, Department of Physics, Athens, Greece

84 National Centre for Nuclear Research, Warsaw, Poland

85 National Institute of Science Education and Research, Homi Bhabha National Institute, Jatni, India

86 National Nuclear Research Center, Baku, Azerbaijan

${ }^{87}$ National Research Centre Kurchatov Institute, Moscow, Russia

88 Niels Bohr Institute, University of Copenhagen, Copenhagen, Denmark

89 Nikhef, National institute for subatomic physics, Amsterdam, Netherlands

90 NRC Kurchatov Institute IHEP, Protvino, Russia

${ }^{91}$ NRNU Moscow Engineering Physics Institute, Moscow, Russia

92 Nuclear Physics Group, STFC Daresbury Laboratory, Daresbury, United Kingdom

93 Nuclear Physics Institute of the Czech Academy of Sciences, Rež u Prahy, Czech Republic

94 Oak Ridge National Laboratory, Oak Ridge, Tennessee, United States

95 Ohio State University, Columbus, Ohio, United States

${ }_{96}$ Petersburg Nuclear Physics Institute, Gatchina, Russia

97 Physics department, Faculty of science, University of Zagreb, Zagreb, Croatia

98 Physics Department, Panjab University, Chandigarh, India

99 Physics Department, University of Jammu, Jammu, India

100 Physics Department, University of Rajasthan, Jaipur, India

101 Physikalisches Institut, Eberhard-Karls-Universität Tübingen, Tübingen, Germany 
118 Nucléaire $(D P h N)$, Saclay, France

136 Università degli Studi di Foggia, Foggia, Italy

137 Università degli Studi di Pavia, Pavia, Italy

138 Università di Brescia, Brescia, Italy

139 Variable Energy Cyclotron Centre, Homi Bhabha National Institute, Kolkata, India

140 Warsaw University of Technology, Warsaw, Poland

141 Wayne State University, Detroit, Michigan, United States

142 Westfälische Wilhelms-Universität Münster, Institut für Kernphysik, Münster, Germany

143 Wigner Research Centre for Physics, Hungarian Academy of Sciences, Budapest, Hungary

144 Yale University, New Haven, Connecticut, United States

145 Yonsei University, Seoul, Republic of Korea 